\title{
ENVOLVENTE DE RESISTENCIA LATERAL DE PISO PARA ESTRUCTURAS DE MAMPOSTERÍA CONFINADA
}

\author{
Sergio Alcocer M..$^{(1)}$, Hugo Hernández B. ${ }^{(2)}$ y Harry Sandoval R. ${ }^{(3)}$
}

\begin{abstract}
RESUMEN
En este artículo se presenta un procedimiento para calcular la curva envolvente de resistencia lateral de estructuras de mampostería confinada con mediana o baja altura. La curva envolvente idealizada se definió con parámetros obtenidos con pruebas experimentales realizadas en México. Las curvas envolventes de resistencia lateral analíticas son comparadas con las curvas experimentales obtenidas en un modelo probado bajo carga cíclica reversible y una serie en modelos de mampostería probados en la mesa vibradora del II-UNAM. Los resultados sugieren una adecuada predicción del límite elástico, resistencia máxima y estado último. El procedimiento incluye el cálculo de las ductilidades local y global y del factor de comportamiento sísmico.
\end{abstract}

Palabras clave: curva envolvente; mampostería confinada; ductilidad; coeficiente sísmico; coeficiente sísmico de desempeño; falla por fuerza lateral; falla por carga vertical

\begin{abstract}
This paper presents a procedure for calculating the envelope curve of lateral resistances of confined masonry structures with medium or low height. The idealized envelope curve of lateral resistance was defined by parameters obtained with experimental tests performed in Mexico. The analytical envelope curves of lateral resistance are compared with experimental curves obtained in a model tested under cyclic reversible load and a series of tests on models of masonry in shaking table of the II-UNAM. Results suggest an adequate prediction of the elastic limit, maximum resistance and ultimate state. The procedure includes the calculation of local and global ductility and seismic behavior factor.
\end{abstract}

Keywords: envelope curve; confined masonry; ductility; seismic coefficient; seismic coefficient of performance; fault lateral force; vertical load fails

\footnotetext{
Artículo recibido el 28 de noviembre de 2011 y aprobado para su publicación el 10 de junio de 2013. Se aceptarán comentarios y/o discusiones hasta cinco meses después de su publicación.

(1) Instituto de Ingeniería de la UNAM, Coordinación de Estructuras y Materiales, Apartado Postal 70-472, México DF, 041510, Tel. (+52 55) 5622-8946. salcocerm@iingen.unam.mx.

(2) Universidad Michoacana de San Nicolás de Hidalgo, Edificio de Posgrado en Ingeniería Civil, CU, Morelia, Michoacán, Tel. (+52 443) 322 3500, ext. 4341, hugohbarrios@yahoo.com.mx

(3) Egresado de la Maestría en Estructuras, Programa de Maestría y Doctorado en Ingeniería, UNAM.
} 


\section{INTRODUCCIÓN}

Existen varias propuestas para modelar analíticamente estructuras de mampostería confinada, éstas se pueden clasificar en: (a) Micro-modelado, (b) Macro-modelado y (c) modelado por medio de curvas envolvente de comportamiento histerético. El micro-modelado de los elementos que forman un muro de mampostería se basa principalmente en técnicas numéricas como el Elemento Finito y Elementos de Frontera (Ishibashi y Kastumata, 1994). Debido a su alto costo computacional y el uso de software especializado e incertidumbre en las propiedades de los materiales, aún no es justificable que dichas metodologías se apliquen de manera práctica.

Actualmente es posible, con cualquier software comercial, modelar estructuras de mampostería con macro-modelos estructurales. Los macro-modelos o elementos estructurales más populares son las barras y puntales a compresión y el método de la columna ancha (Holmes, 1961; Kadir, 1974; Chrysostomou et al., 1992; Mosalam et al., 1996; Madan et al., 1997; Crisafulli, 1997; Tomazevic, 1999). También se han propuesto algunas modificaciones a dichos elementos (Beyer et al., 2008; Zúñiga y Terán, 2008). En México, Bazán (1980) demostró la capacidad de la metodología de la columna ancha para representar adecuadamente la rigidez lateral elástica de muros de mampostería confinada sujetos a cargas laterales. En Zúñiga y Terán (2008) se propone una modificación a dicho modelo con el fin de estimar la respuesta no lineal de estructuras de mampostería, para lo cual calculan curvas de capacidad y umbrales de desplazamientos asociados a los estados límite. Aun cuando los autores encuentran una elevada variabilidad entre los resultados experimentales estudiados, consideran que la metodología que proponen para calcular las curvas de capacidad de los especímenes ofrece estimaciones razonablemente conservadoras, y que la resistencia asociada al primer agrietamiento como la rigidez elástica se obtiene con suficiente precisión (figura 1a).

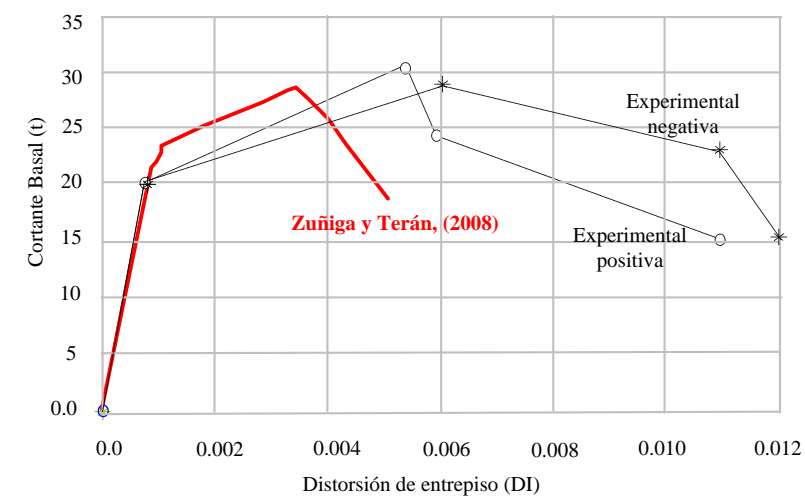

(a) Curvas de capacidad, modelo W-W

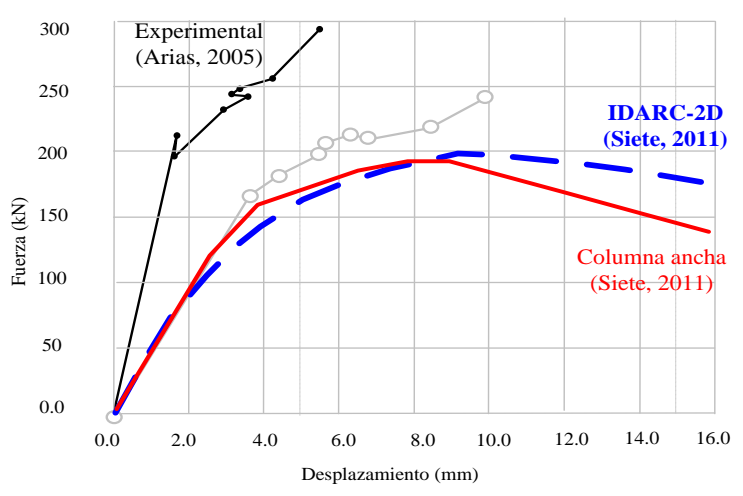

(b) Curvas de capacidad nivel N1, M3SRCC

Figura 1. Curvas de capacidad calculadas con el método de la columna ancha

En otro trabajo (Siete, 2011) se propone calcular curvas de fragilidad validando las curvas de capacidad calculadas con el método de la columna ancha ya que el método de puntales a compresión, usando el software IDARC-2D (Valles et al., 1996) no predice adecuadamente los resultados experimentales. El argumento del autor es que la curva calculada con el método de la columna ancha captura adecuadamente el comportamiento inicial en rigidez y resistencia, pero después del agrietamiento las curvas se separan de los resultados experimentales (figura 1b). Alcocer et al., (2004) al comprar los valores experimentales en mesa vibradora con los obtenidos con un modelo simple, obtuvieron buenos resultados en cuanto al cortante basal pero encontraron ciertas diferencias en los ángulos de distorsión asociados, por lo que sugieren mejorar el modelado no lineal de la mampostería confinada, principalmente 
con el fin de capturar adecuadamente el confinamiento especialmente para grandes ángulos de distorsión. Esto también se evidencia en la figura 2, en donde para una estructura denominada 3D (Sánchez et al., 2010) se comparan las curvas envolventes analíticas calculadas por diferentes autores y metodologías, con la curva envolvente experimental. Las gráficas indican que las diversas metodologías predicen adecuadamente la pendiente inicial que representa el comportamiento elástico del muro, pero todas difieren después de este punto.

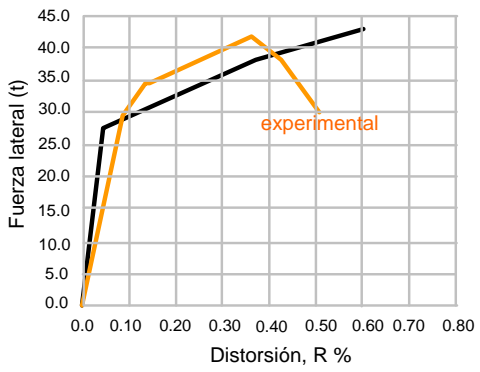

(a) Coral (2004)

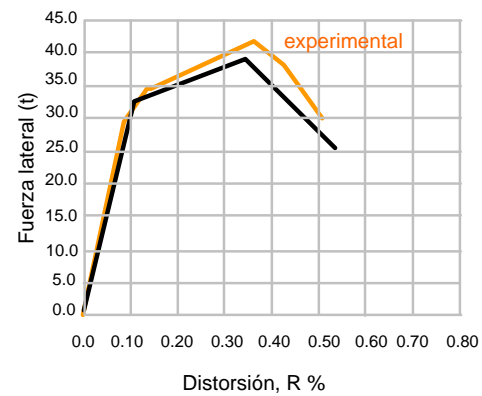

(b) Zúñiga y Terán (2008)

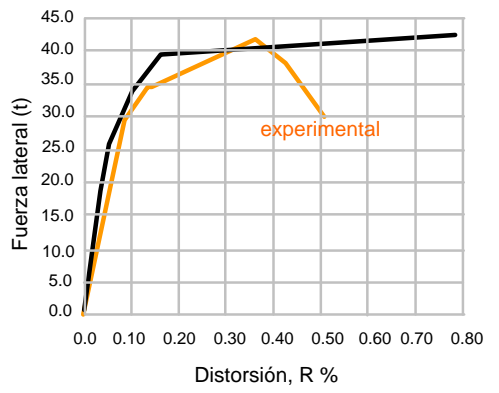

(c) Sulpicio, et al. (2010)

Figura 2. Envolvente Positiva, estructura 3D

En cuanto a los modelos analíticos basados en una curva envolvente de comportamiento histerético de muros de mampostería, Meli (1979) propuso una curva trilineal que relaciona el esfuerzo cortante con la distorsión asociada. Moroni, Astroza y Tavonatti (1994) a partir de estudios experimentales realizados en estructuras de mampostería confinada, propusieron un modelo trilineal para caracterizar el comportamiento no-lineal de la mampostería ante cargas laterales. Flores (1995) con base en los resultados experimentales obtenidos en el Centro Nacional de Prevención de Desastres (CENAPRED) propuso un modelo analítico para representar la rigidez de los lazos de histéresis en los ciclos de carga y descarga. El modelo de Flores se basa en la teoría de fricción y es similar al de Meli (1979) excepto que el tercer tramo de la curva considera degradación de resistencia. Tomazevic propuso representar el comportamiento histerético de muros de mampostería sujetos a carga lateral en combinación con carga vertical usando curvas envolventes de tipo trilineal (Tomazevic y Klemenc, 1997a) y bilineal (Tomazevic, 1999). En la figura 3 se muestran esquemáticamente dichas curvas, los valores que las definen se pueden encontrar en las referencias antes citadas.

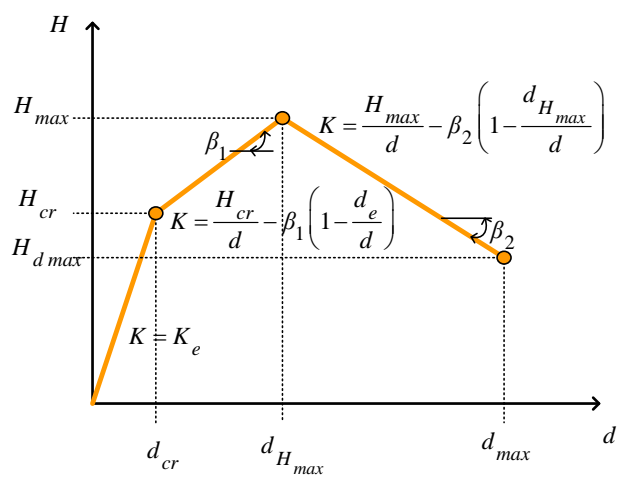

(a) Curva trilineal (Tomazevic y Klemenc, 1997a)

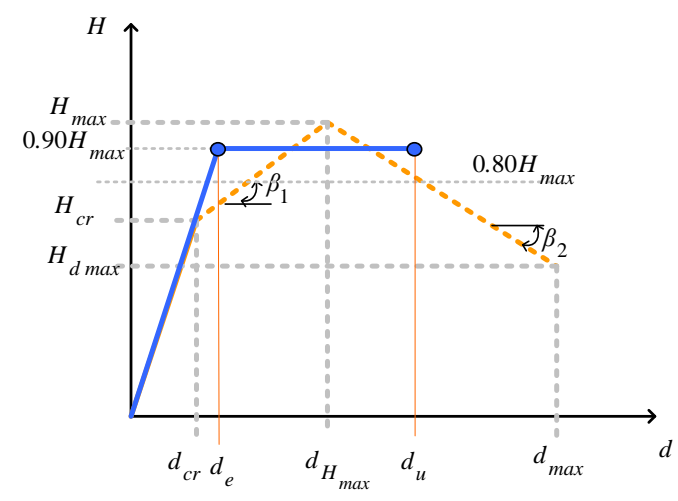

(b) Curva bilineal (Tomazevic, 1999)

Figura 3. Idealización de la envolvente de comportamiento 
Las recomendaciones propuestas en Jean y Cesín (2000) corroboran que el método de la columna ancha es adecuado para el análisis y diseño elástico de estructuras de mampostería confinada y/o reforzada interiormente. De hecho, el método de la columna ancha es usado por Tena et al. (2010) para validar el procedimiento de diseño conocido como Método Simplificado. De lo comentado anteriormente se puede aceptar que el uso de la metodología de la columna ancha es adecuado para el cálculo de la rigidez elástica incluyendo deformaciones por cortante, pero para el cálculo de curvas de capacidad de estructuras de mampostería presenta inconsistencias, principalmente después de que ocurre el primer agrietamiento.

\section{ENVOLVENTE DE RESISTENCIA LATERAL DE PISO PROPUESTA}

La curva de capacidad de una estructura se obtiene a partir de un análisis estático no-lineal bajo desplazamientos laterales monótonamente crecientes. Durante el análisis se aplica una distribución de cargas laterales que mantienen un valor relativo o proporcional a la altura de la estructura pero varían en magnitud. Dicha magnitud se incrementa hasta que la estructura alcanza el desplazamiento lateral asociado a un estado máximo de utilidad que por lo general es la falla o colapso de la estructura. A cada incremento de desplazamiento lateral global de la estructura está asociada una fuerza cortante basal, que graficada versus los desplazamientos produce la curva de capacidad.

Considerando las condiciones de restricción de apoyo de los muros es posible calcular la resistencia a flexión y cortante, así como la rigidez inicial efectiva de todos los muros que forman la estructura. Utilizando una curva idealizada de envolvente del comportamiento histerético se puede obtener la contribución de cada muro a la resistencia del entrepiso para calcular la curva envolvente total (figura 4a). La curva envolvente de entrepiso es la relación entre la resistencia y el desplazamiento relativo del entrepiso, y se obtiene por superposición de las envolventes de resistencia de todos los muros en el entrepiso en consideración (figura 4b). Esta curva proporciona información sobre el comportamiento y nivel de daño estructural, resistencia máxima y colapso de la estructura, y una vez determinada es posible asociar un índice de daño uniforme en la estructura según se muestra en la figura 4b (Fischinger et al., 2004).

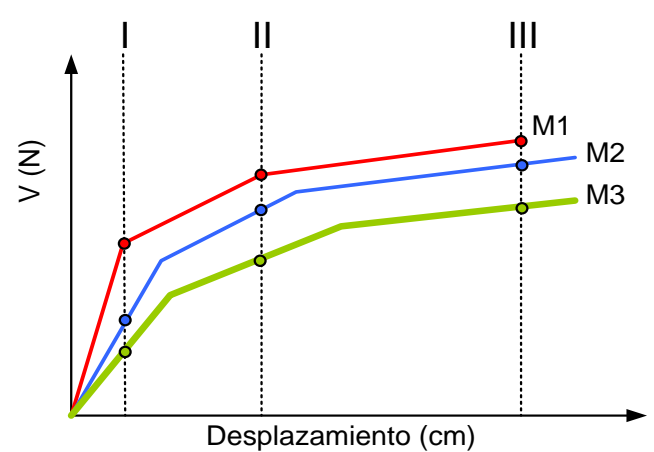

(a) Curvas individuales de cada muro

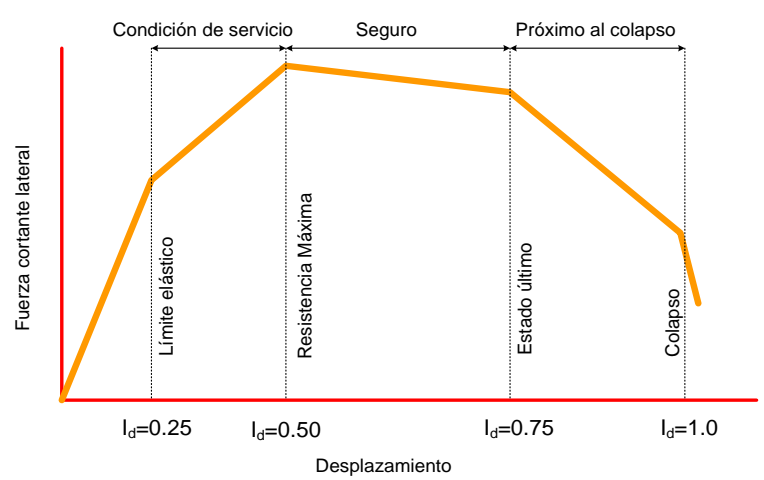

(b) Curva primaria de entrepiso

Figura 4. Concepto de curva primaria de entrepiso

La curva envolvente de resistencia (figura 5) define tres estados límites característicos: (A) Límite Elástico, cuando un muro en el entrepiso alcanza el límite elástico y se agrieta; (B) Estado de Resistencia Máxima; y (C) Estado de Resistencia Última, donde la resistencia del entrepiso se deteriora sobrepasando un límite aceptable establecido. Para fines prácticos, una degradación mayor al $70 \%$ de la resistencia máxima define el estado de colapso de la estructura. 

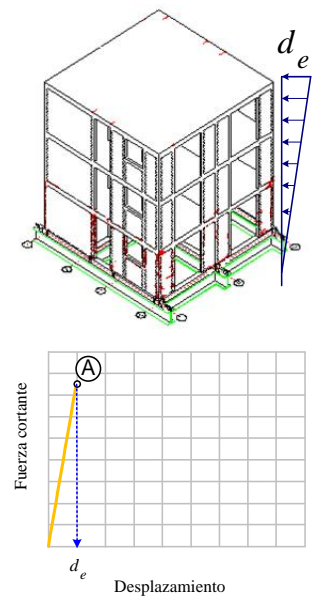

(a) Límite Elástico
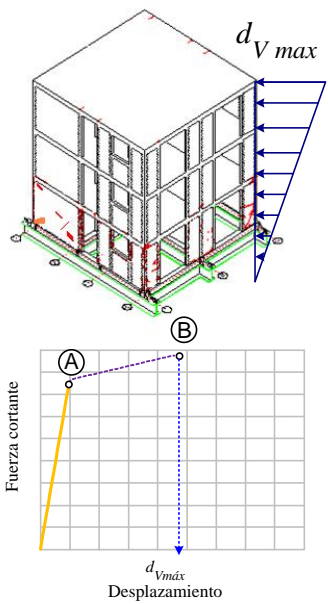

(b) Resistencia máxima
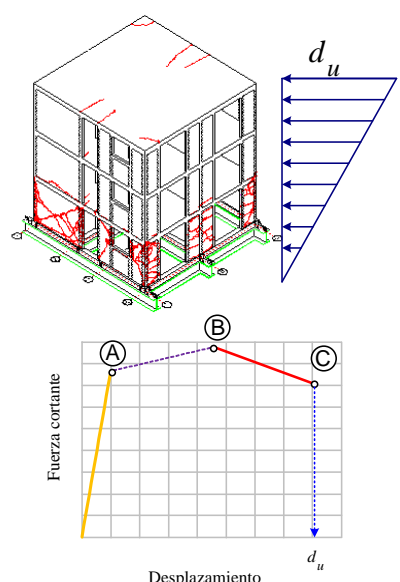

(c) Resistencia última

Figura 5. Caracterización del incremento de desplazamiento en la curva envolvente

En este trabajo al desarrollar las curvas de capacidad se considera que del origen al punto (A) todos los muros están en el intervalo elástico, hasta que se alcanza el punto (B) en el que algún muro llega a su carga máxima. El punto (C) es en donde se considera que la estructura ha alcanzado la falla global.

El procedimiento aquí descrito se emplea para el cálculo de la curva evolvente de resistencia de un entrepiso que forma parte de una estructura de mampostería y que cumple con los requisitos básicos para que sea aplicable el Método de Simplificado de diseño (NTCM-RCDF, 2004). Dicho método se basa en la distribución de fuerzas laterales en estructuras simétricas con diafragmas rígidos cuando la carga lateral se aplica en una sola dirección (Tena et al., 2010).

Con base en los resultados experimentales realizados en modelos de estructuras de mampostería ensayados en mesa vibradora (Tomazevic, 1999; Vázquez, 2005; Barragán, 2005; Arias, 2005) se ha observado que el primer modo de vibrar en cortante prevalece en la respuesta dinámica. Debido a la acción de la losa de entrepiso que funciona como diafragma horizontal rígido, el daño se concentra principalmente en los elementos estructurales del piso inferior, lo anterior se ha observado tanto en pruebas experimentales como después de la ocurrencia de un sismo. 


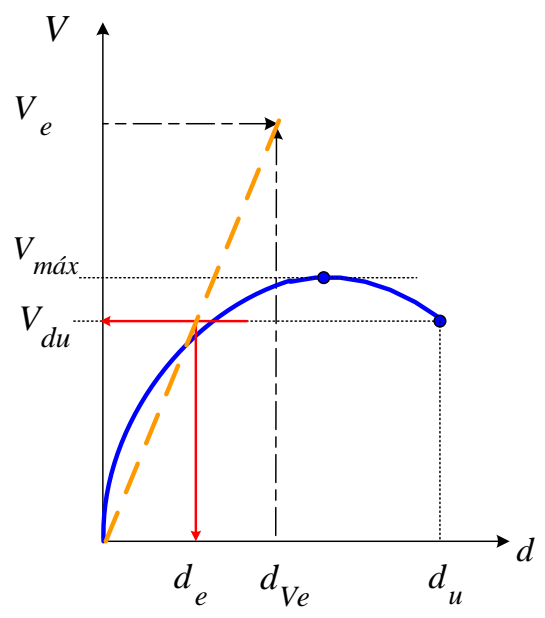

(a) Factor de comportamiento sísmico

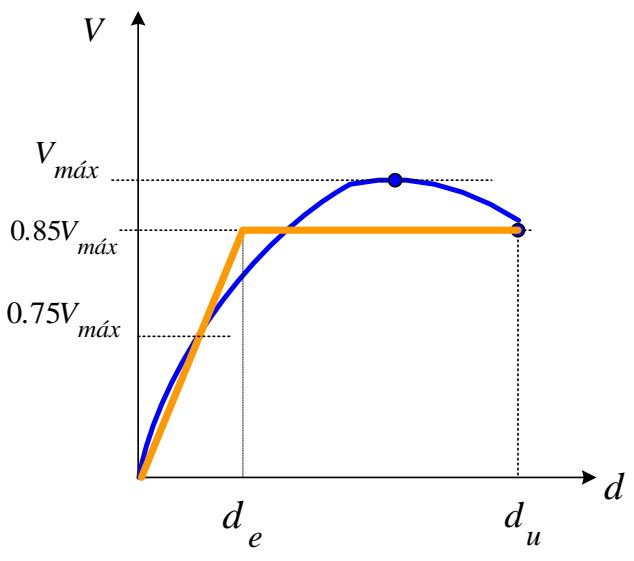

(b) Criterio de ductilidad equivalente

Figura 6. Curvas para definir los términos de ductilidad

Algunos parámetros que definen el comportamiento sísmico de estructuras de mampostería pueden determinarse si se establece la relación entre la resistencia de entrepiso y su correspondiente desplazamiento, es decir, si se determina la curva envolvente de resistencia de entrepiso (figura 6a) y además se determina el factor de comportamiento sísmico, $Q$, definido (Tomazevic et al., 2004) como la relación entre la fuerza lateral que produce el colapso de la estructura y la fuerza que causa la iniciación del daño, es decir, el primer cambio en la rigidez estructural,

$$
Q=\frac{V_{e}}{V_{d u}},
$$

donde $V_{e}$ es la fuerza elástica y $V_{d u}$ es la carga última de diseño. Para el caso de estructuras de mampostería confinada de piezas macizas se recomienda un valor de $Q=2$ (NTCS-RCDF, 2004). Aunque el coeficiente sísmico no sólo depende de la ductilidad sino también de la energía histerética, para estructuras relativamente rígidas (Bazán, 1980; Newmark y Hall, 1982; Tomazevic, 1999) una forma aproximada de obtener este parámetro es:

$$
Q=\sqrt{2 \mu_{u}-1}
$$

donde $\mu_{u}$ es el factor de ductilidad global. Para estructuras de mampostería confinada considerando el valor de $Q=2$ en la ecuación 2 se tiene que $\mu_{u}=2.5$. Para sistemas estructurales en donde la falla se concentra en la planta baja, como las que se estudian en este trabajo, la ductilidad global y la del primer nivel se relacionan (Paulay y Priestley, 1992) con:

$$
\mu_{1}=1+\frac{2}{3} n\left(\mu_{u}-1\right)
$$

donde $\mu_{1}$ es la ductilidad en la planta baja y $n$ es el número de niveles de la estructura. El término $2 / 3$ que aparece en la ecuación (3) supone que el desplazamiento debido a la fluencia, en el primer nivel de 
una estructura, es aproximadamente dos terceras partes del desplazamiento total de la estructura (Paulay y Priestley, 1992). Como en este trabajo se calcularan las envolventes de resistencia de la planta baja de la estructura, será necesario calcular la ductilidad global conocida la ductilidad del primer entrepiso,

$$
\mu_{u}=\frac{3}{2 n}\left(\mu_{1}-1\right)+1
$$

Una vez calculada la curva envolvente de resistencia del nivel 1, la ductilidad, $\mu_{1}$, se obtiene con el criterio de ductilidad equivalente (Priestley, Seible y Calvi, 1996), en donde la respuesta se idealiza según una curva bilineal equivalente (figura $6 \mathrm{~b}$ ),

$$
\mu_{1}=\frac{d_{u}}{d_{e}}
$$

en donde $d_{u}$ es el desplazamiento último equivalente y $d_{e}$ es el desplazamiento idealizado de diseño. Este criterio se basa en la determinación de una rigidez inicial secante al $75 \%$ de la carga última, la cual a su vez se define como la asociada al 15\% de degradación de resistencia.

\section{CURVA ENVOLVENTE IDEALIZADA}

En la figura 7 se muestra una curva típica del comportamiento histerético obtenida experimentalmente en un muro de mampostería sujeto a cargas laterales cíclicas y que relaciona la carga lateral medida con sus respectivos desplazamientos laterales. Las curvas envolventes de resistencia varían según sea el tipo de mampostería, tal que la mampostería confinada por dalas y castillos tiene un mejor comportamiento que la mampostería no confinada. El comportamiento de la mampostería por lo general se caracteriza por las deformaciones por cortante que son las que dominan. En una curva idealizada (Tomazevic y Klemenc, 1997a) que represente la envolvente de resistencia de un muro se identifican tres zonas principales: (a) Zona de comportamiento elástico, en la que se presenta agrietamiento horizontal en los castillos y agrietamiento mínimo en los elementos de mampostería, en esta zona se puede decir que se presenta un comportamiento prácticamente elástico asociado con una rigidez lateral efectiva, $K_{e}$; (b) Zona de máxima resistencia, que está asociada a una carga lateral máxima, $V_{\text {máx }}$, y su respectivo desplazamiento, $d_{V m a ́ x}$. En esta zona la rigidez del muro, $K_{V m a ́ x}$, es menor que la rigidez anterior al primer agrietamiento, lo que le permite alcanzar la máxima resistencia; y (c) Zona de degradación de rigidez y de resistencia, definida por la carga lateral que produce el desplazamiento último o justo antes del colapso. 


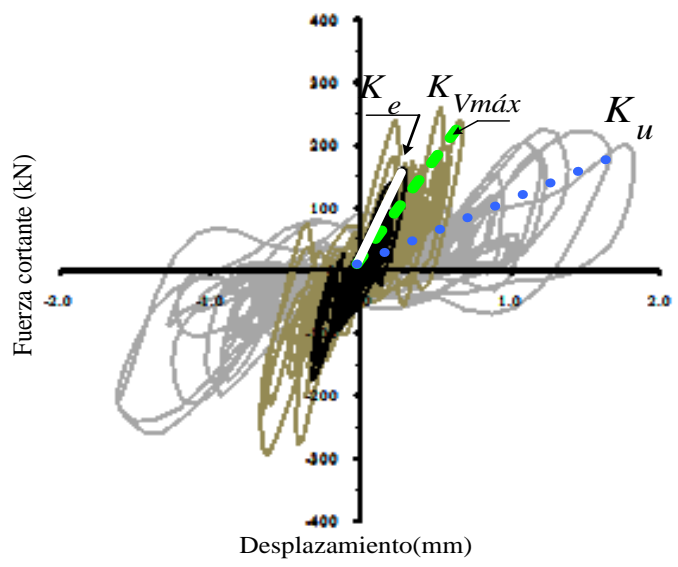

Figura 7. Curva de histéresis típica de un muro de mampostería ante cargas laterales cíclicas

Con base en las tres zonas descritas se propone la curva envolvente del comportamiento idealizada mostrada en la figura 8. La fuerza cortante resistente de diseño especificada en las NTCM-RCDF (2004) se establece como la fuerza cortante correspondiente al primer agrietamiento diagonal de la mampostería, $V_{\text {agr }}$ :

$V_{\text {agr }}=\left(0.5 v_{m}^{*}+0.3 \sigma\right) A_{T} \leq 1.5 v_{m}^{*} A_{T}=V_{n N T C}$,

donde $v_{m}^{*}$ es la resistencia a compresión diagonal de la mampostería, $\sigma$ es el esfuerzo de compresión y $A_{T}$ es el área transversal del muro, incluidos los castillos, sin transformar su área. La mampostería no se comporta elásticamente, ni siquiera para pequeñas deformaciones. Sin embargo, la rigidez elástica efectiva, $K_{e}$, se puede definir como la pendiente de la primer recta de la curva idealizada,

$K_{e}=\frac{V_{a g r}}{d_{a g r}}$,

donde $d_{a g r}$ es el desplazamiento asociado a la primera grieta significante en el muro. En muros sin refuerzo transversal la resistencia máxima a cortante se alcanza después de que se ha generalizado el agrietamiento inclinado. La resistencia en este punto será la suma de la resistencia al corte de los castillos y la contribución de la mampostería agrietada. En general, cuando se presenta el cortante máximo en el muro, la grieta inclinada ha penetrado los castillos. En Flores (1996) se propone que la fuerza cortante máxima se puede estimar como:

$V_{\text {máx }}=\eta V_{d}+V_{a g r}$,

donde $\eta=0.30$ es un factor de eficiencia y $V_{d}$ representa la contribución del refuerzo vertical por acción de dovela. Dulacska, Flores y Alcocer (2001) proponen: 
$V_{d}=1.26 d_{b}^{2} \sqrt{f_{c}^{\prime} f_{y}}$,

donde $d_{b}$ es el diámetro de las varillas; $f_{c}^{\prime}$ y $f_{y}$ son el esfuerzo a compresión del concreto y de fluencia del acero de refuerzo, respectivamente. Considerando que este valor es proporcional a la fuerza cortante de agrietamiento se tiene,

$\eta V_{d}=x V_{a g r}$.

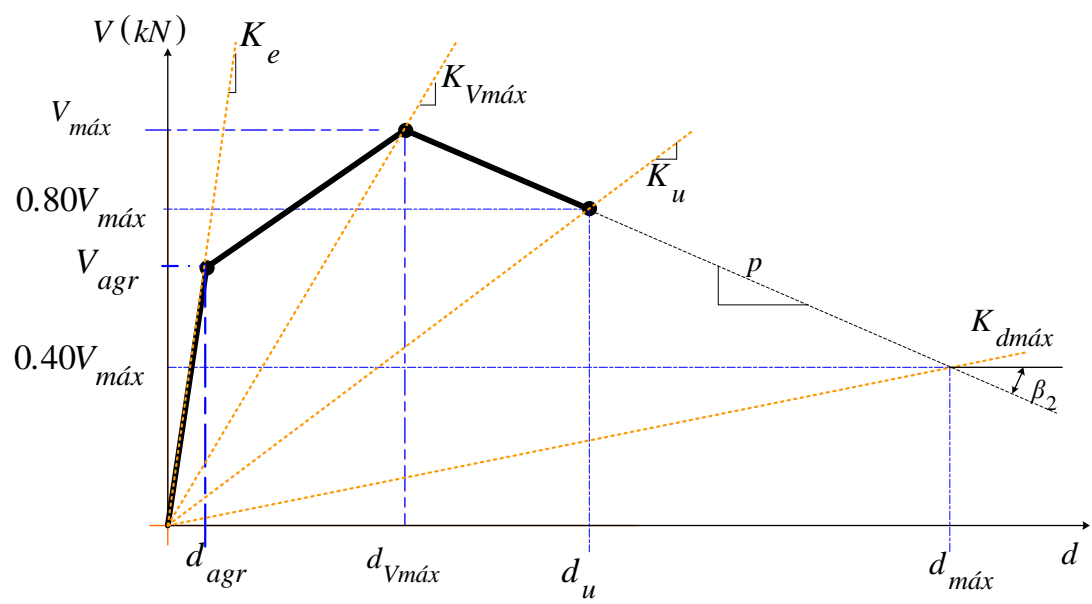

Figura 8. Curva envolvente idealizada

Sustituyendo la ecuación 10 en la 8 se puede escribir,

$V_{\text {máx }}=(1+x) V_{\text {agr }}$,

donde $x$ vale 0.25 (Flores y Alcocer, 2001). Después del agrietamiento, el muro tiene una rigidez secante definida como la relación entre su resistencia lateral y su correspondiente desplazamiento. La rigidez asociada a la fuerza cortante máxima se puede definir como:

$K_{\text {Vmáx }}=\frac{V_{\text {máx }}}{d_{\text {Vmáx }}}=\frac{(1+x) V_{a g r}}{d_{\text {Vmáx }}}$.

Sustituyendo el cortante asociado al agrietamiento obtenido con la ecuación 7 en la 12 se tiene,

$\frac{K_{\text {Vmáx }}}{K_{e}}=\frac{(1+x) d_{a g r}}{d_{\text {Vmáx }}}$. 
El grado de degradación de la rigidez secante depende del daño, de la correlación del índice de daño y de la rigidez secante del muro. Tomazevic (1999) considera que la relación de rigideces varía entre 0.32 y 0.42 . En este trabajo se considera que,

$$
\frac{K_{V m a ́ x}}{K_{e}}=0.25,
$$

por lo que el desplazamiento asociado es:

$$
d_{\text {Vmáx }}=4(1+x) d_{\text {agr }}
$$

El desplazamiento máximo se presenta para una fuerza cortante de,

$$
V_{d m a ́ x}=0.40 V_{\text {máx }}
$$

tal que la relación de rigideces es:

$$
\frac{K_{d m a ́ x}}{K_{e}}=0.03
$$

y por tanto el desplazamiento máximo se presenta en:

$$
d_{\text {máx }}=13.33(1+x) d_{\text {agr }} \text {. }
$$

La recta que une los puntos de desplazamiento máximo y cortante máximo, tiene una pendiente negativa con magnitud:

$$
\beta_{2}=0.0643 K_{e}
$$

y el desplazamiento último (figura 8) es:

$$
d_{u}=d V_{\text {máx }}+\frac{0.2 V_{\text {máx }}}{\beta_{2}},
$$

es decir,

$$
d_{u}=7.11(x+1) d_{a g r}
$$

El punto en donde se presenta la resistencia última no corresponde al cortante de diseño, sino a un valor máximo experimental idealizado. Tomazevic (1999) encontró que el valor obtenido experimentalmente en más de 60 muros es: 
$V_{u}=0.80 V_{\text {máx }}$

y la rigidez secante asociada es:

$K_{u}=\frac{V_{u}}{d_{u}}=\frac{0.8(1+x) V_{a g r}}{d u}$

para calcular el desplazamiento último, $d_{u}$, se considera que la relación entre rigideces tangentes para esta zona es:

$\frac{K_{u}}{K_{e}}=0.1125$

El índice de degradación de rigidez lateral (Anónimo, 1999) se puede escribir en forma aproximada como,

$\frac{K}{K_{e}}=\frac{1}{\left(1+5300 R^{1.20)}\right.}$

donde $R$ es la distorsión lateral del muro. Considerando como correcta esta ecuación, para una distorsión lateral $R=0.002$, la cual controla el estado límite de resistencia (Astroza y Schmidt, 2004), se tiene:

$$
\frac{K_{V \operatorname{máx}}}{K_{e}}=0.25
$$

que coincide con el valor considerado en este trabajo (ecuación 14). Y para una distorsión lateral de $R=0.0044$, que controla el estado límite último (Astroza y Schmidt, 2004),

$$
\frac{K_{u}}{K_{e}}=0.1126
$$

que es igual a la propuesta en este trabajo en la ecuación 24. De esta manera, el modelo de la curva envolvente de histéresis refleja matemáticamente los resultados experimentales obtenidos en México. En la figura 9 se observa la curva envolvente propuesta, la relación entre los desplazamientos y la distorsión del muro, daño asociado, y su relación con los Estados Límite propuestos por Astroza y Schmidt (2004). 


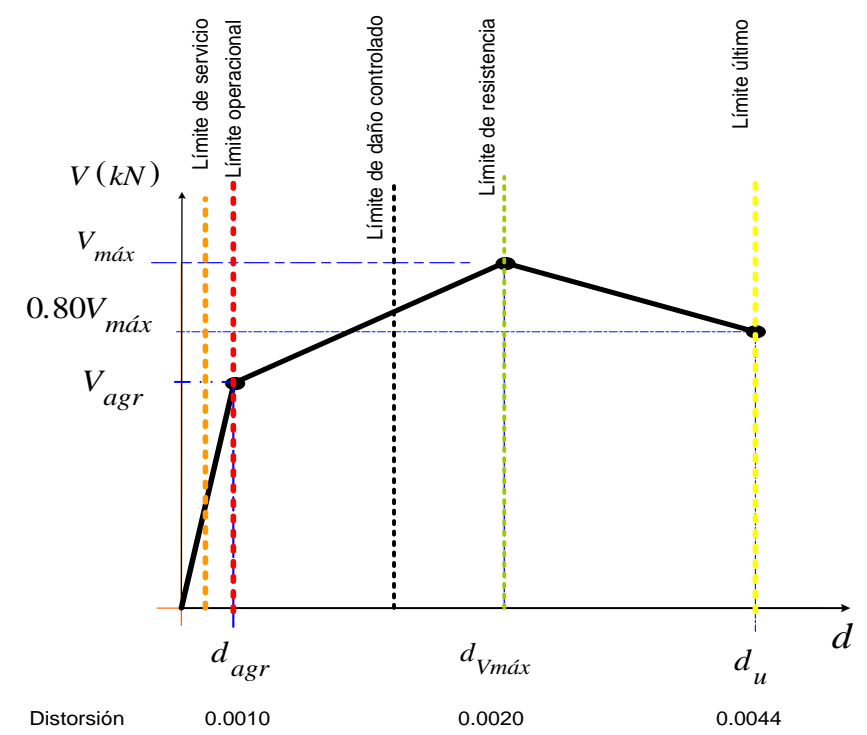

Figura 9. Relación de desplazamientos, distorsión, daño y Estados Límite

\section{HIPÓTESIS DE CÁLCULO PARA LA CURVA ENVOLVENTE DE ENTREPISO}

Se realizan las siguientes hipótesis para el cálculo de la curva envolvente:

(1) Las losas de piso trabajan como diafragmas horizontales rígidos, tal que los desplazamientos en cada uno de los muros del entrepiso i-ésimo, $D_{i}$, debido a la fuerza cortante aplicada en el entrepiso, $V_{i}$, se distribuyen proporcionalmente a la rigidez lateral de cada muro, $k_{j i}$.

(2) Los desplazamientos debidos a los efectos por torsión son proporcionales a la rigidez del muro.

(3) Predomina el primer modo de vibrar. Se imponen desplazamientos horizontales que siguen la forma modal del primer modo (figura 10b).

(4) La contribución individual de los muros para resistir la fuerza lateral del entrepiso dependen de los desplazamientos laterales tomados por el muro y de la forma de la curva envolvente idealizada de resistencia del muro. Los muros resisten los desplazamientos impuestos hasta agotar su capacidad de ductilidad individual, aunque pueden fallar por carga lateral, todavía son capaces de resistir carga vertical. (5) En los muros con forma de "T", " $L$ " o en forma de cruz, se considera que los patines están desligados verticalmente del alma.

(6) Las aberturas como puertas y ventanas mantienen un esquema uniforme en elevación. 


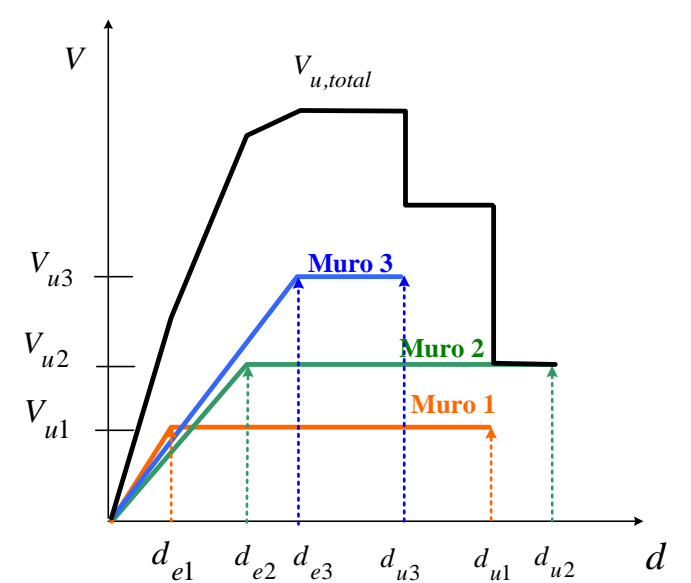

(a) Contribución de cada muro en la curva envolvente total

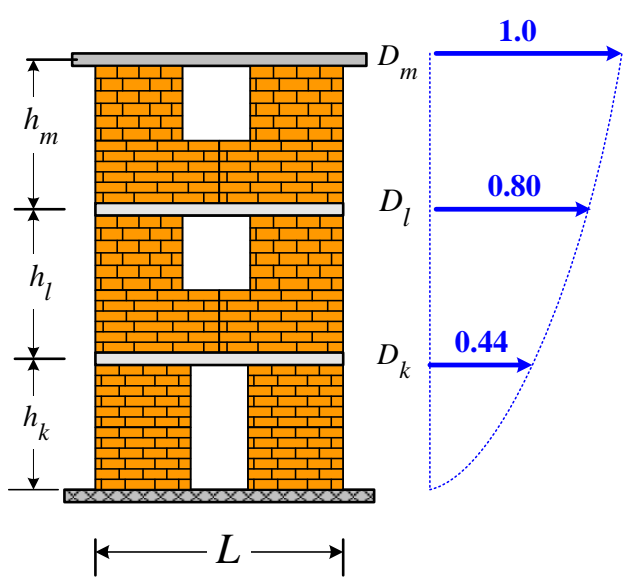

(b) Distribución de desplazamientos

Figura 10. Hipótesis para el cálculo de la curva envolvente de resistencia

\section{PROCEDIMIENTO DE CÁLCULO}

La metodología propuesta para calcular la curva envolvente de resistencia de entrepiso es iterativa y programable en una hoja de cálculo, el procedimiento se inicia con:

(1) Se calculan las constantes de cálculo del análisis como son: (a) el módulo de elasticidad de la mampostería y del concreto; (b) el módulo de rigidez al corte y compresión diagonal de la mampostería; (c) la masa de cada nivel considerando los entrepisos a ejes medios en la altura y (d) los esfuerzos a compresión actuantes en cada muro.

(2) Se calcula de la rigidez lateral efectiva de cada muro y la fuerza cortante resistente. En este trabajo, la rigidez lateral efectiva para cada muro se calculó con (UNIANDES, 2001):

$$
K_{e}=\left[\frac{h^{3}}{\beta E_{m} I_{t}}+\frac{\gamma h}{A G_{m}}\right]^{-1},
$$

donde $h$ es la altura efectiva del muro, $E_{m}$ es el módulo de elasticidad de la mampostería, $G_{m}$ es el módulo de rigidez al corte, $A$ es el área efectiva al cortante, $\beta$ es un factor que depende las condiciones de apoyo en los extremos del muro y que se considera igual a 3 para muros en voladizo y de 12 para muros doblemente empotrados, en este trabajo se consideró $\beta=12.0$. El valor del factor correctivo, $\gamma$, es de 1.2 para muros con sección rectangular, aunque puede usarse un valor de $\gamma=1.0$ si los muros tienen castillos en sus extremos que actúan como patines; en este trabajo se consideró $\gamma=1.2$. El momento de inercia de la sección transformada, $I_{t}$, se puede calcular con:

$$
I_{t}=I_{m}+I_{a}+I_{c}
$$

donde $I_{m}$ es el momento de inercia de la mampostería confinada, 


$$
I_{m}=\frac{t L^{3}}{12}
$$

donde $t$ es la espesor de la mampostería y $L$ es la longitud del muro. La contribución del acero al momento de inercia, $I_{a}$, se puede obtener con:

$$
I_{a}=n_{s}\left[A_{s 1} d_{s 1}^{2}+A_{s 2} d_{s 2}^{2}\right]
$$

y la contribución del concreto que forma los castillos, $I_{c}$, con:

$$
I_{c}=n_{c} t_{c}\left[\frac{b_{1}^{3}}{12}+\frac{b_{2}^{3}}{12}+b_{1} d_{c 1}^{2}+b_{2} d_{c 2}^{2}\right]
$$

donde $A_{s 1}$ y $A_{s 2}$ son el área de acero que forma los castillos, $d_{s i}$ y $d_{c i}$ son las distancias del centroide del muro al centroide del acero y al centroide del castillo, respectivamente; $b_{i}$ y $t_{c}$ son el ancho y el espesor de los castillos. Las relaciones entre los módulos de elasticidad de la mampostería con el acero de refuerzo, $E_{s}$, y con el concreto que forma los castillos, $E_{c}$, están definidas respectivamente por:

$$
n_{s}=\frac{E_{s}}{E_{m}} \text { y } n_{c}=\frac{E_{c}}{E_{m}} .
$$

(3) Se forma la matriz de rigidez lateral de los muros que forman los entrepisos, la cual se puede aproximar mediante un modelo de cortante despreciando las deformaciones por flexión que presentan los muros.

(4) Para considerar los efectos por torsión (Chipol, 2001) se calculan: (a) la posición del centro de masas (CM); (b) el centro de rigidez (CR); (c) la excentricidad; (d) el momento debido a torsión; (e) la rigidez torsional y (f) los desplazamientos debidos a la torsión. El sistema estructural debe satisfacer la fórmula aproximada propuesta en el método simplificado de diseño (NTCM-RCDF, 2004) para calcular la excentricidad estática en el nivel i-ésimo, $e_{s i}$, que se toma como la distancia entre centroide de las áreas efectivas de los muros con respecto al centro de cortante. La excentricidad estática calculada debe ser,

$e_{s i} \leq 0.10 B_{i}$

$B_{i}$ es la dimensión perpendicular del entrepiso medida en dirección del análisis (figura 11). 


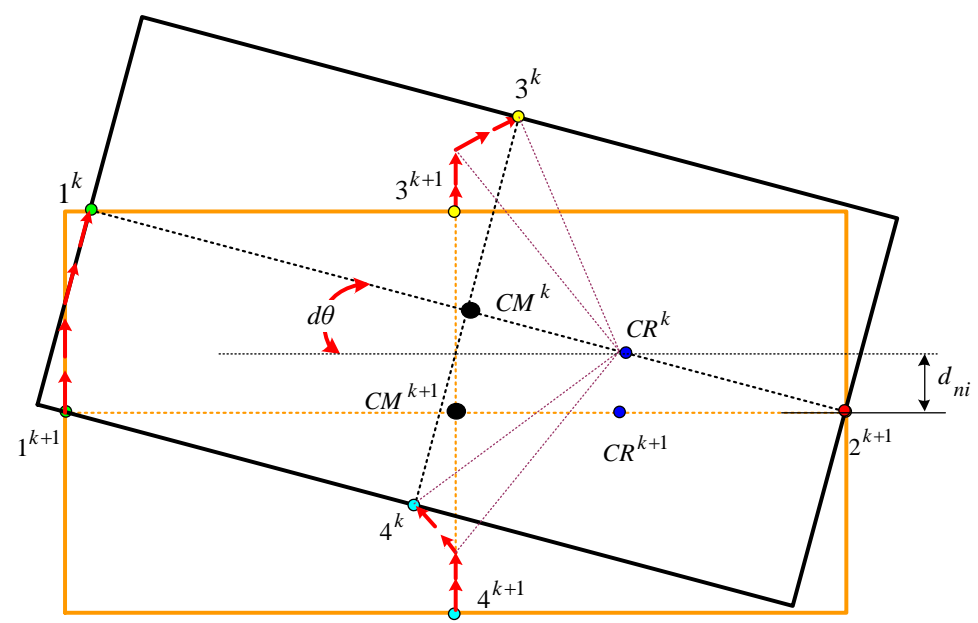

Figura 11. Posición del centro de masas y de rigidez, debida a efectos por torsión

(5) Se realiza la sumatoria de la fuerza cortante y de la rigidez de los muros en el sentido del análisis por nivel.

(6) Se calculan los desplazamientos, incluyendo los referentes a torsión.

(7) Se calcula la forma modal del primer modo de vibrar con cualquier metodología de dinámica estructural. En este trabajo se utilizó el método iterativo del vector inverso (Chopra, 1995).

(8) Se revisa el criterio de falla. Si la estructura aún no alcanza la falla se impone un desplazamiento arbitrario en el último nivel y los cálculos se repiten, regresando al paso (2).

(9) Se calcula la ductilidad y el factor de comportamiento sísmico.

(10) Finaliza el procedimiento.

El diagrama de flujo del procedimiento anteriormente descrito se muestra en forma esquemática en las figuras 12 y 13 . 


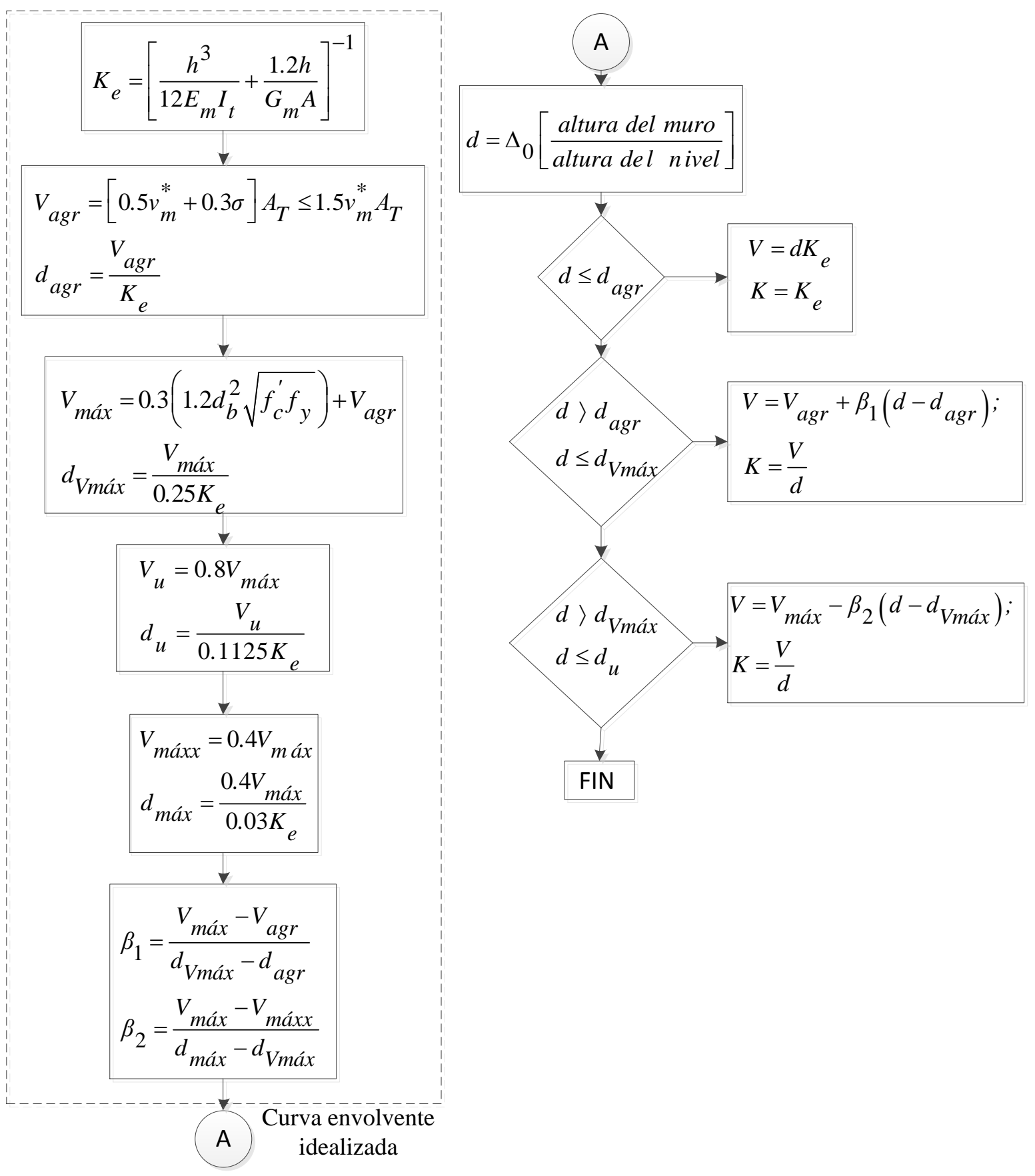

Figura 12. Diagrama de Flujo para el cálculo de la fuerza cortante resistente y de la rigidez 

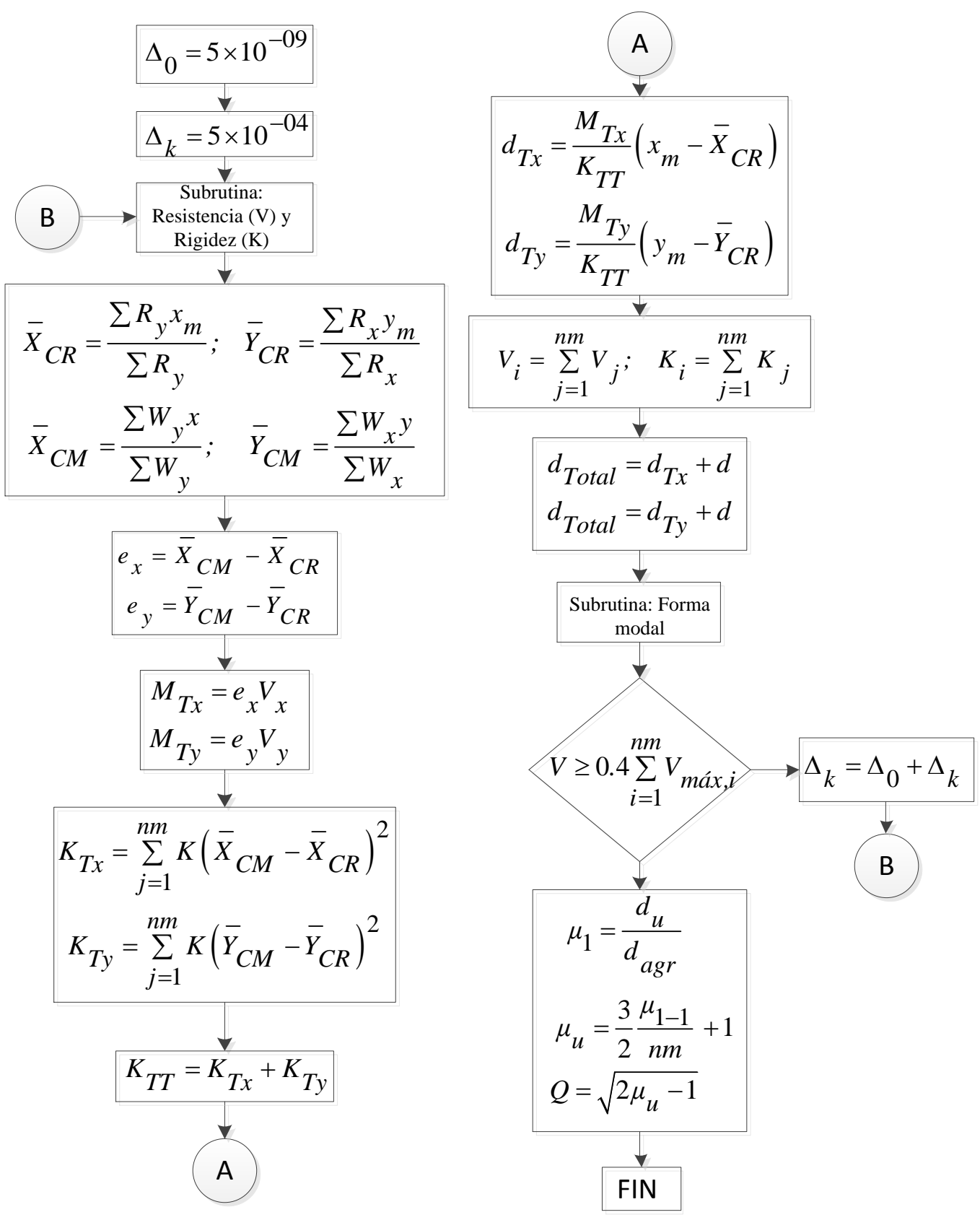

Figura 13. Diagrama de Flujo para el procedimiento general de cálculo de la curva envolvente de resistencia lateral de piso para estructuras de mampostería confinada

\section{CALIBRACIÓN DEL PROCEDIMIENTO}

Con el fin de comprobar el procedimiento de cálculo de las curvas envolventes de resistencia lateral de piso, se calcularon las curvas envolventes de los modelos reportados en Tomazevic y Klemenc (1997b) usando la curva trilineal propuesta por dichos autores. Los modelos corresponden a una estructura de tres niveles de mampostería confinada y probados en una mesa vibradora. Al modelo de la estructura probada 
en la dirección longitudinal es M1 y en la dirección transversal M2. En la figura 14 se comparan las curvas envolventes de resistencia del primer nivel calculadas analíticamente en este trabajo con las reportadas experimentalmente en dicha referencia. En las figuras 14a y $14 \mathrm{~b}$ se observa que los valores de la curva analítica que definen la rama ascendente, el cortante máximo, la fuerza cortante en la que se presenta el primer agrietamiento así como el desplazamiento que lo acompaña se compara bien con los valores obtenidos experimentalmente. Las curvas analíticas calculadas en este trabajo difieren a lo más en $5 \%$ con respecto a las analíticas reportadas en Tomazevic y Klemenc (1997b), atribuible a la diferencia de valores tomados como referencia en las curvas trilineales.

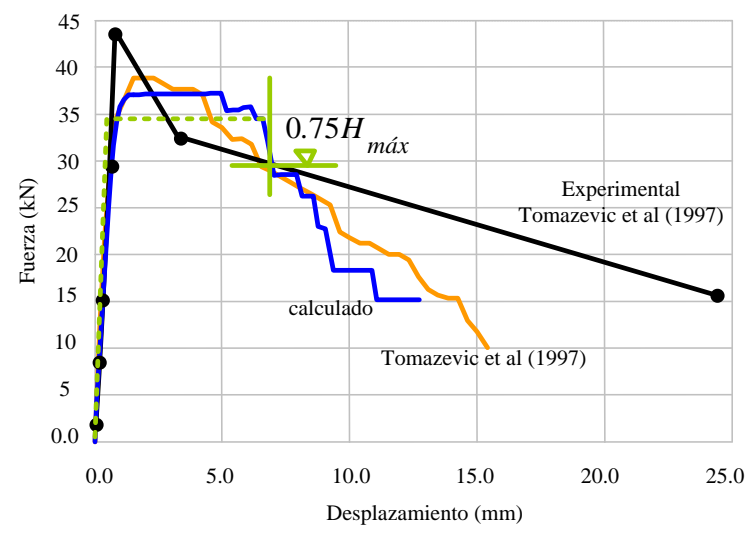

(a) Modelo M1, dirección longitudinal

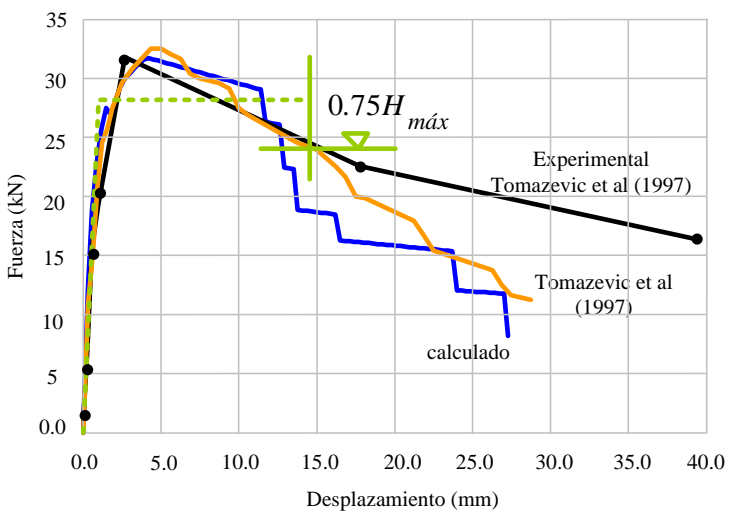

(b) Modelo M2, dirección transversal

Figura 14. Comparación entre curvas envolventes, experimentales y analíticas

En la Tabla 1 se resumen los valores de ductilidad global y local, así como el del factor de comportamiento sísmico, calculados analíticamente y los obtenidos con los valores experimentales de Tomazevic y Klemenc (1997b). Además, en la última columna se listan los valores del factor de comportamiento sísmico, $Q$, calculado analíticamente por dichos autores. Se ve que tanto la ductilidad como los del factor de comportamiento sísmico predichos por el modelo son adecuados.

Tabla 1. Comparación de valores de ductilidad

\begin{tabular}{|l|c|c|c|c|c|c|c|}
\hline \multirow{2}{*}{ MODELO } & \multicolumn{2}{|c|}{$\mu_{1}$} & \multicolumn{2}{c|}{$\mu_{u}$} & \multicolumn{3}{c|}{$Q$} \\
\cline { 2 - 8 } & experimental & analítico & experimental & analítico & experimental & analítico & $\begin{array}{c}\text { Tomazevic } \\
\text { y Klemenc } \\
\text { (1997) }\end{array}$ \\
\hline M1 & 6.27 & 7.84 & 3.64 & 4.42 & 2.50 & 2.80 & 2.91 \\
M2 & 11.56 & 9.59 & 6.28 & 5.30 & 3.40 & 3.10 & 2.47 \\
\hline
\end{tabular}

\section{COMPARACIÓN CON RESULTADOS EXPERIMENTALES REALIZADOS EN MÉXICO}

Para comparar el procedimiento y el modelo de la curva envolvente propuesta, se compararon las curvas envolventes de resistencia de piso obtenidas analíticamente con las experimentales en una serie de modelos ensayados en la mesa vibradora del Instituto de Ingeniería de la UNAM, II-UNAM (Vázquez, 2005; Barragán, 2005; Arias, 2005) y con la obtenida en el ensayo con carga cíclica reversible del modelo 3D (Sánchez, 1998). Los datos referentes a dimensiones y propiedades de los materiales empleados en los modelos se especifican en las referencias antes citadas. 
El primer modelo ensayado en la mesa vibradora representa un edificio de un nivel (Vázquez, 2005). La distribución en planta de los muros del modelo inicial, M1SRSC se muestra en la figura 15a y su vista en elevación en la figura 15b. Debido a las características mecánicas de la mampostería usada y a la configuración estructural, el modelo resultó con rigidez y resistencia elevada, por lo que debió ser sometido a diversos ciclos de carga. En la figura 16a se muestra la comparación de los valores experimentales con la curva envolvente calculada con el procedimiento propuesto en este trabajo. Se comprueba que el modelo se encontraba lejos de la zona del límite elástico durante la prueba.

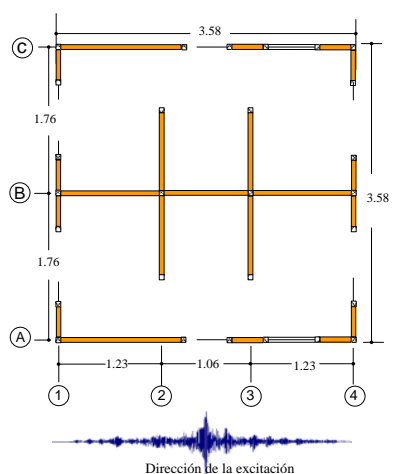

(a) Modelo M1SRSC

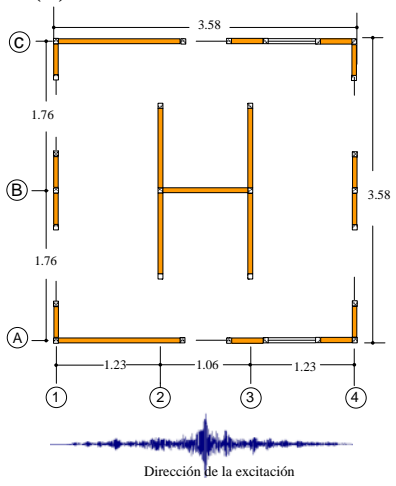

(c) Modelo M1-M

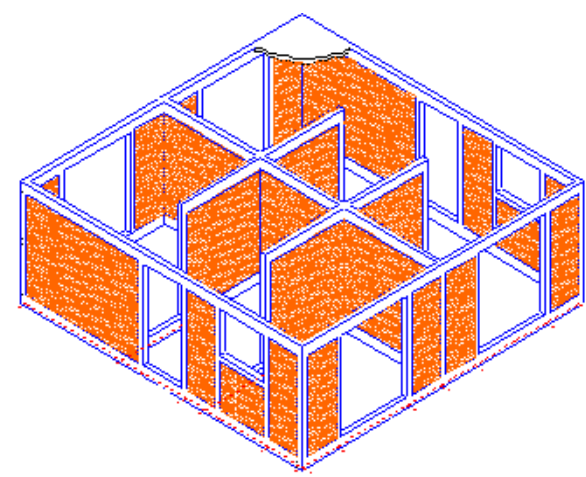

(b) Vista general

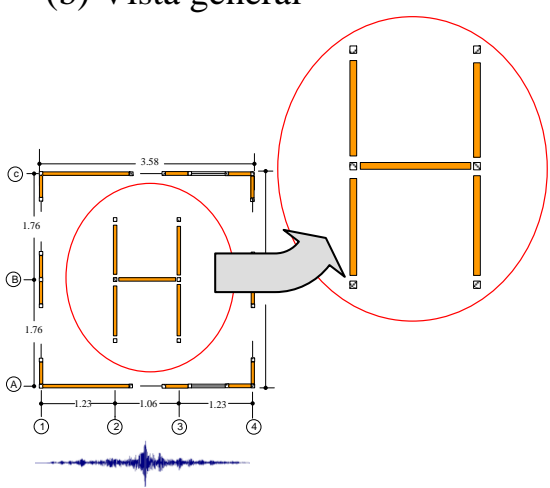

(d) Modelo M1-A

Figura 15. Descripción de los modelos de un nivel (Vázquez, 2005), el análisis se realizó en la dirección de los ejes letras

Para continuar con las pruebas experimentales se modificó el modelo M1SRSC en su distribución lateral. La primera modificación que se realizó fue retirar dos de los muros centrales, y se llamó M1-M (figura 15c). En la figura 16b se muestran las curvas envolventes obtenidas analítica y experimentalmente. La prueba dinámica se suspendió cuando se presentó el primer agrietamiento, punto que coincide con el obtenido con la curva analítica. 


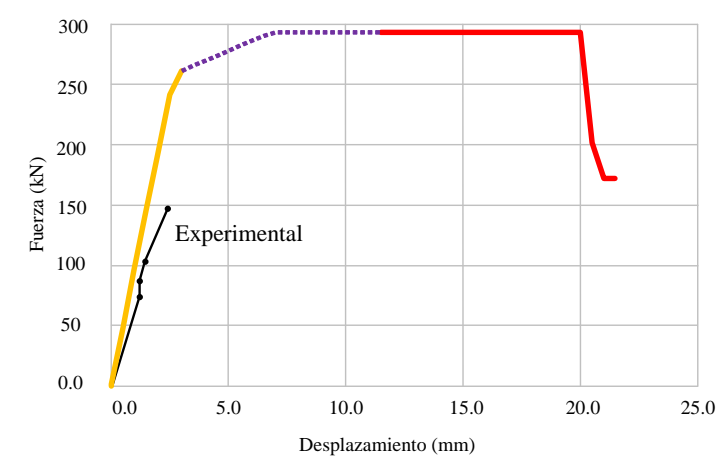

(a) Modelo M1SRSC

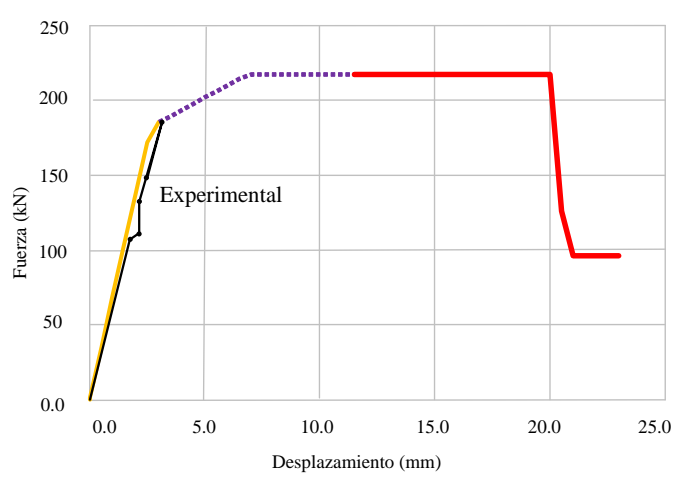

(b) Modelo M1-M

Figura 16. Curvas envolventes de resistencia y valores experimentales

La segunda modificación al modelo fue separar verticalmente los muros centrales (figura 15d); a este modelo se le llamó M1-A. La separación de los muros centrales modificó ligeramente la curva experimental; la prueba se suspendió cuando ocurrió el primer agrietamiento, este punto lo predice adecuadamente la curva envolvente (figura 17a).

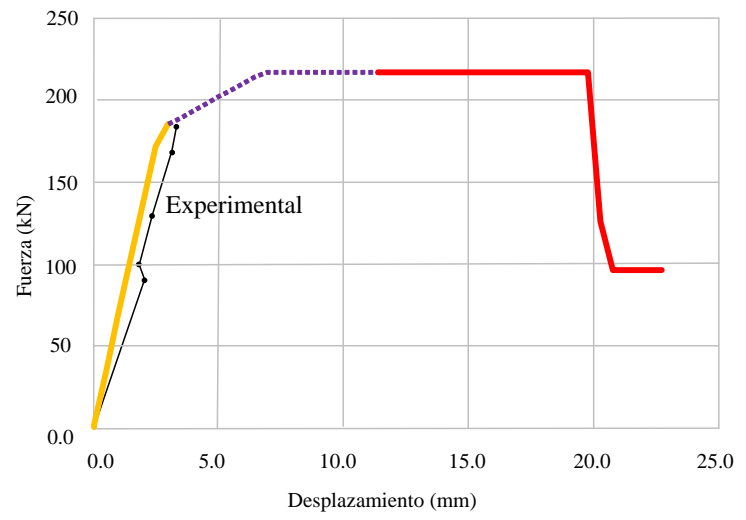

(a) Modelo M1-A

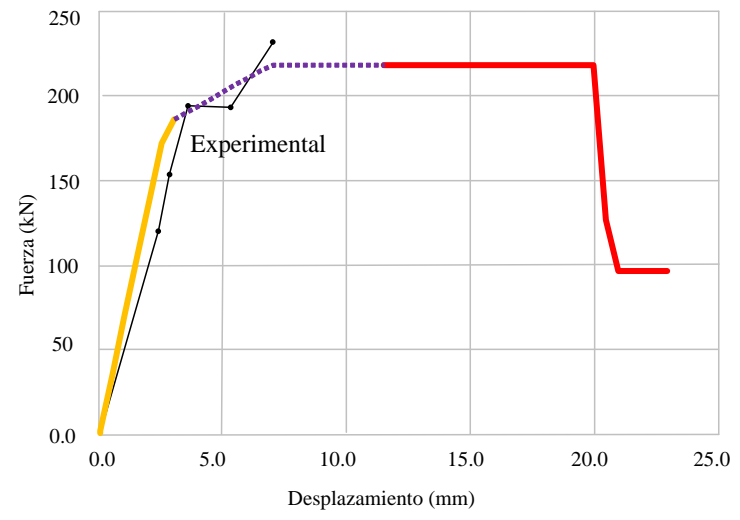

(b) Modelo M1-B

Figura 17. Curvas envolventes de resistencia y valores experimentales

La tercera modificación fue colocar en el modelo M1-A un peso de $15.7 \mathrm{kN}$ (1600 kgf) y recibió el nombre de M1-B. La curva envolvente y los valores experimentales se muestran en la figura 17b. Se puede ver que aparentemente la carga máxima en la que se suspendió la prueba resultó mayor que el valor máximo que cubre la curva envolvente; esto se explica más adelante. 


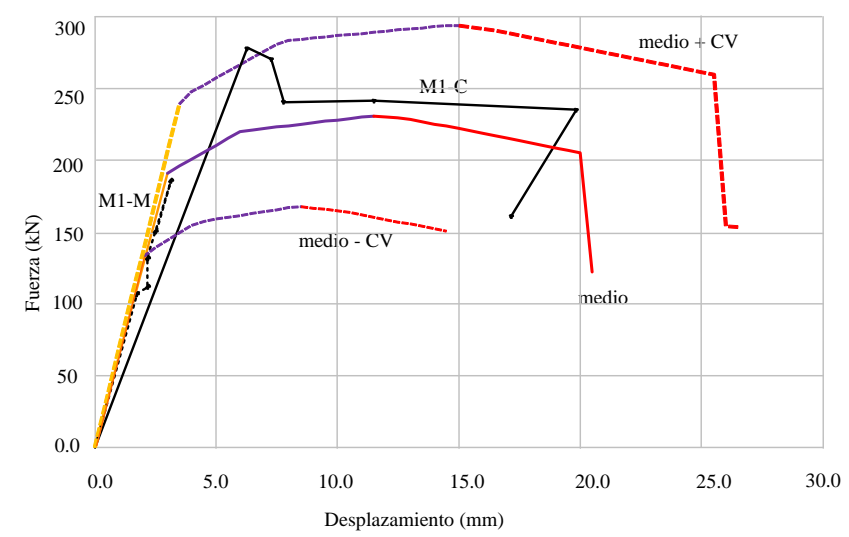

(a) Influencia del cortante resistente

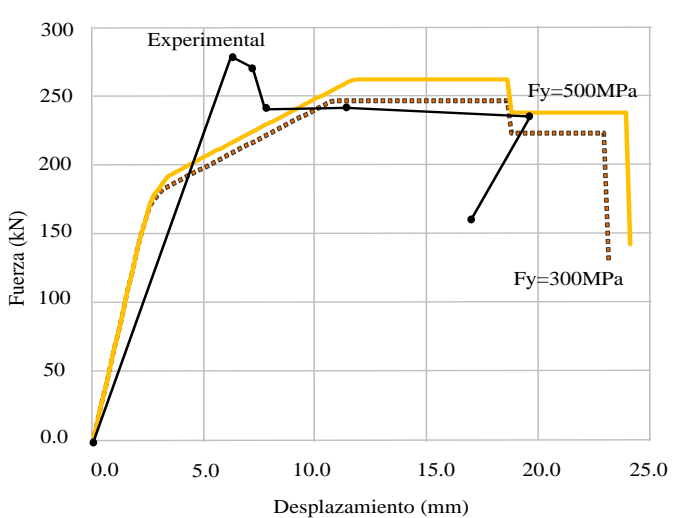

(b) Influencia de la resistencia del acero

Figura 18. Curvas envolventes de resistencia y valores experimentales, modelo M1-C

La cuarta modificación al modelo consistió en colocar sobre el modelo M1-A un peso de $20.6 \mathrm{kN}$ (2100 kgf) y recibió en nombre de M1-C. En la figura 18a se muestra la curva envolvente y los valores experimentales obtenidos en este modelo. Debido a que el modelo M1-C presentaba agrietamiento importante en el momento de la prueba la curva envolvente y los valores experimentales se separan en la rama ascendente, lo cual no pasa al comparar en la misma figura los valores del modelo M1-M. También se observa que la carga máxima experimental es mucho mayor que la que se predice en forma analítica usando un módulo de cortante promedio. Sin embargo, los valores del módulo de cortante de la mampostería utilizada en el modelo presentan un coeficiente de variación (CV) de 0.39. Si se calculan las curvas envolventes con el valor medio más el CV y el valor medio menos el CV, se observa la influencia de este parámetro sobre la forma y magnitud de las cargas resistentes (figura 18a). Para este caso la curva calculada con el valor de esfuerzo promedio más el CV, envuelve a los datos experimentales. El esfuerzo de fluencia del acero de refuerzo utilizado en los castillos durante la prueba también presentó variaciones importantes, pero como se observa en la figura $18 \mathrm{~b}$, no tiene una influencia importante en la curva envolvente. En este trabajo se utilizó un valor de $440 \mathrm{MPa}$ que es el valor medio del acero utilizado en la prueba.

En la segunda serie de estructuras de mampostería probadas en la mesa vibradora del II-UNAM, se modeló una estructura de dos niveles (Barragán, 2005) con la misma estructuración y características de los materiales empleados en el modelo de un nivel, M1-M, descrito en la figura 15c. Dicho modelo recibió el nombre de M2SRCC y se muestra en la figura 19a. La curva envolvente de resistencia del primer nivel junto con los resultados experimentales se presentan en la figura 19b. En este caso la curva envolvente se calculó considerando:

$$
G_{m}=0.20 E_{m} \text { y } v_{m}^{*}=\sqrt{f_{m}^{\prime}}
$$

donde $f_{m}^{\prime}\left(\mathrm{kg}_{\mathrm{f}} / \mathrm{cm}^{2}\right)$ es el esfuerzo a compresión de la mampostería. 


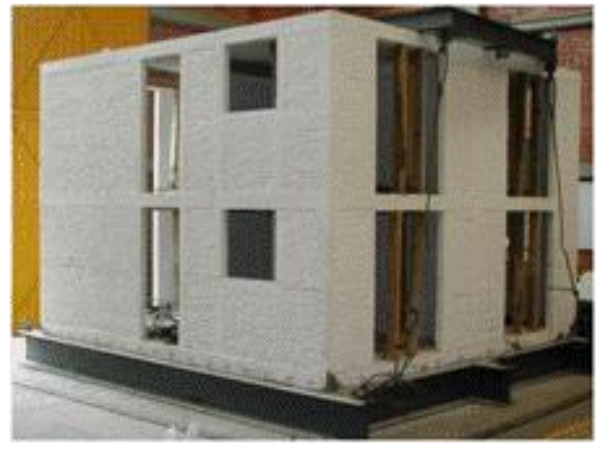

(a) Vista general del modelo

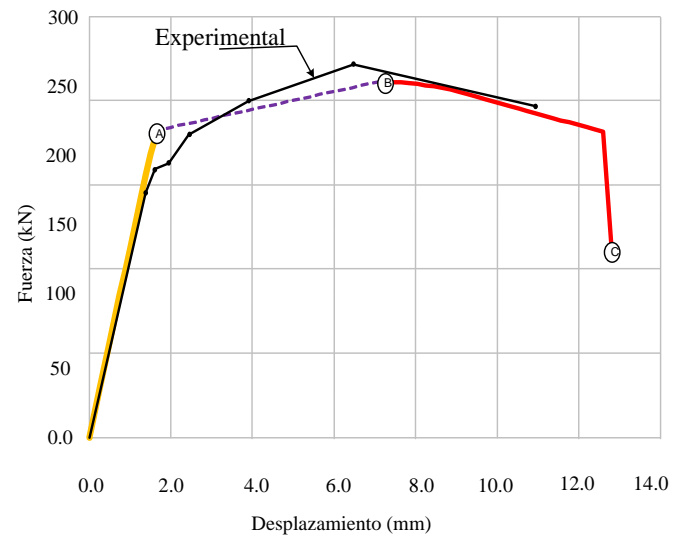

(b) Curva analítica y resultados experimentales

Figura 19. Descripción del modelo de dos niveles, M2SRCC (Barragán, 2005)

No fue posible analizar el desplazamiento que se presenta en la carga lateral última ya que las pruebas experimentales se suspendieron cuando se aplicó una fuerza del $89 \%$ de la resistencia máxima. Sin embargo, se corrobora que el modelo y el procedimiento para calcular la curva envolvente de respuesta predicen adecuadamente la carga del primer agrietamiento diagonal, la carga máxima y sus respectivos desplazamientos.

Como se observa en la figura 20 el tercer modelo de mampostería ensayado en la mesa vibradora del II-UNAM, representa una estructura de tres niveles (Arias, 2005) y tenía similares características mecánicas de los materiales empleados en los anteriores modelos: M1SRSC y M2SRCC. El modelo fue ensayado con dos configuraciones diferentes: el M3SRCC con una distribución de muros similar al modelo M1SRSC (figura 20a) y el modelo M3-M sin los muros centrales (figura 20b).

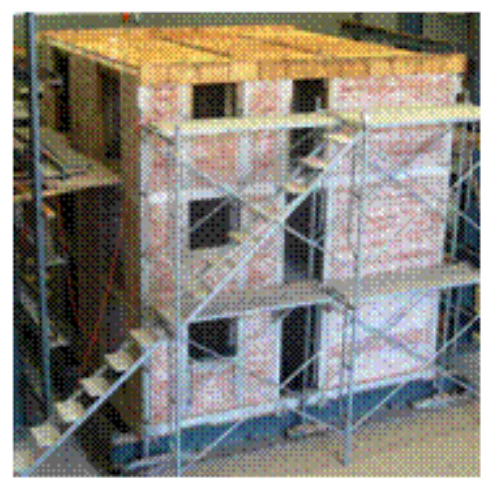

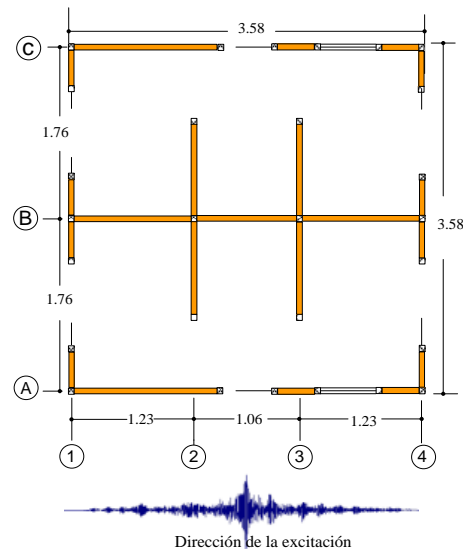

(a) Modelo M3SRCC

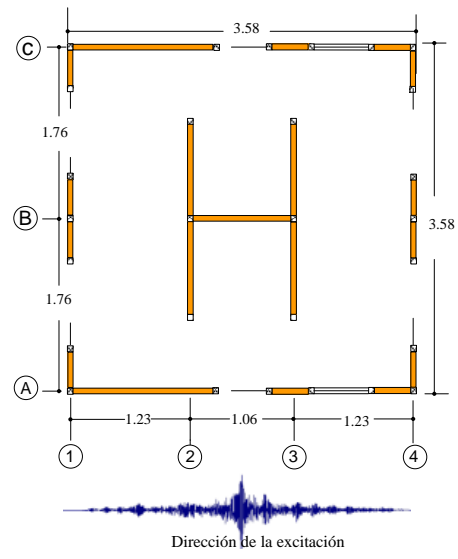

(b) Modelo M3-M

Figura 20. Descripción de los modelos con tres niveles (Arias, 2005)

Las curvas envolventes de resistencia del primer nivel se calcularon con los valores considerados anteriormente. Las pruebas experimentales del modelo M3SRCC se suspendieron antes de que se presentara degradación de resistencia, por lo que los valores experimentales sólo son comparables con los 
analíticos en la rama ascendente (figura 21a). Se encontró que los valores analíticos calculados en este trabajo se ajustan mejor con los experimentales que los calculados con la metodología de la columna ancha (figura 1b). Los datos experimentales obtenidos con el modelo M3-M no son comparables con los resultados analíticos debido a que cuando se ensayó este modelo se encontraba agrietado debido a ensayos anteriores (figura 21b).

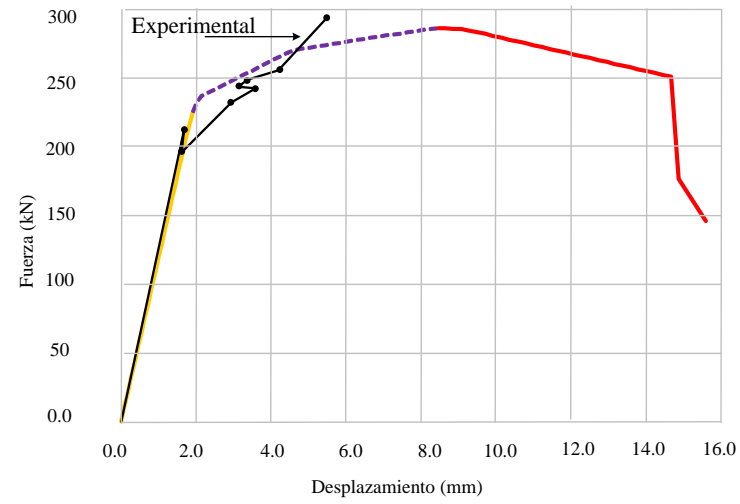

(a) M3SRCC

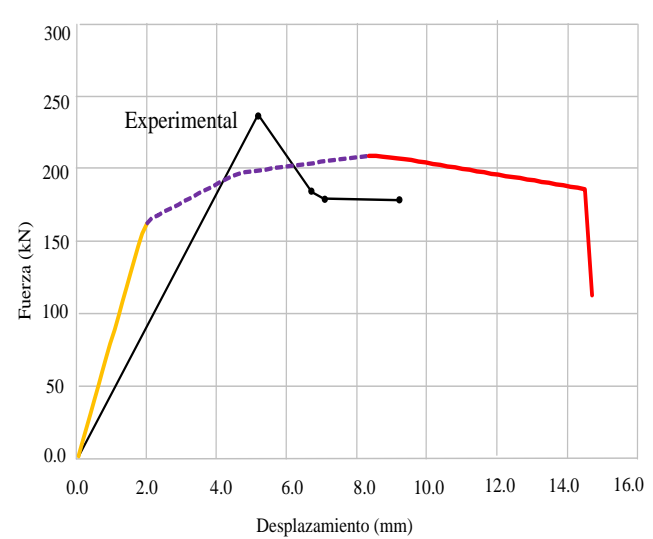

(b) M3-M

Figura 21. Curvas envolventes de resistencia del primer nivel, modelo con tres niveles

El cuarto modelo es el ensayado en el Laboratorio de Estructuras Grandes del CENAPRED, con carga cíclica reversible y una configuración en planta ideal y simétrica (Sánchez, 1998). Este modelo es llamado 3D (figura 22a) y consistió de dos niveles a escala 1:1 con mampostería confinada. La curva envolvente de resistencia del primer nivel y los resultados experimentales se muestran en la figura 22b. Se observa que la curva envolvente predice muy bien la carga lateral máxima y su desplazamiento asociado; además del cortante de agrietamiento, aunque no el desplazamiento en el que se presenta. En este caso no fue posible verificar el desplazamiento a la carga última ya que el modelo no se llevó al colapso debido a que durante el trabajo experimental se planeaba repararlo.

Los resultados descritos en los párrafos precedentes corroboran que son adecuados los valores propuestos para la curva envolvente idealizada y la validez del procedimiento propuesto para el cálculo de la curva envolvente de resistencia lateral de entrepiso.

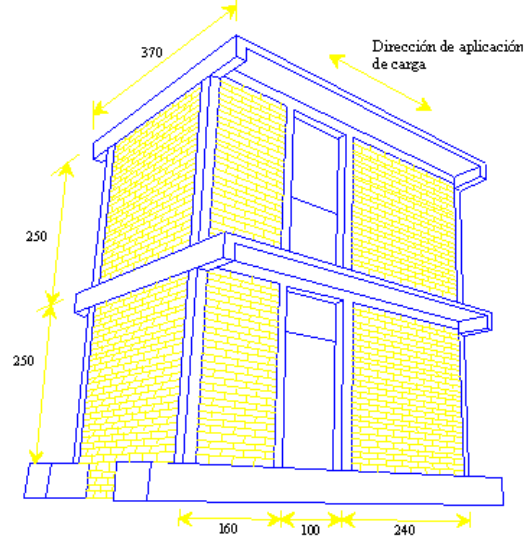

(a) Elevación del modelo 3D

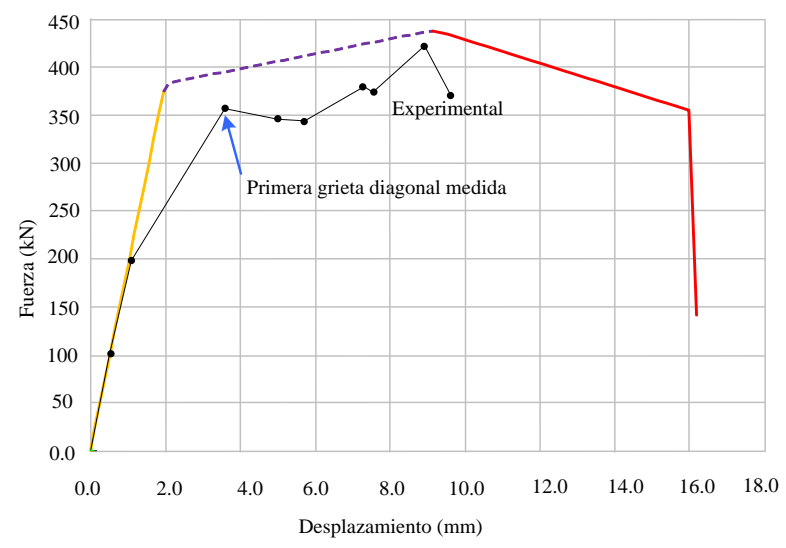

(b) Curva envolvente de resistencia, modelo 3D

Figura 22. Descripción del modelo de dos niveles (Sánchez, 1998) 
En la Tabla 2 se registran los valores de las ductilidades de entrepiso y global, así como del factor de comportamiento sísmico, calculados con la metodología desarrollada en este trabajo. En todos los casos el factor de comportamiento sísmico es mayor que el prescrito por la NTCM-RCDF (2004).

Tabla 2. Valores de ductilidad calculada analíticamente

\begin{tabular}{|l|l|l|l|}
\hline \multicolumn{1}{|c|}{ MODELO } & $\mu_{1}$ & $\mu_{u}$ & $Q$ \\
\hline M1SRSC & 6.15 & 6.15 & 3.36 \\
M1-M & 6.35 & 6.35 & 3.42 \\
M1-A & 6.35 & 6.35 & 3.42 \\
M1-B & 6.35 & 6.35 & 3.42 \\
M1-C & 6.01 & 6.01 & 3.32 \\
M2SRCC & 6.72 & 5.29 & 3.08 \\
M3SRCC & 6.14 & 3.57 & 2.48 \\
M3-M & 5.95 & 3.48 & 2.44 \\
3D-CENAPRED & 6.44 & 5.08 & 3.02 \\
\hline
\end{tabular}

\section{APLICACIÓN A UNA ESTRUCTURA HIPOTÉTICA}

En este apartado se aplicará el procedimiento propuesto para calcular la curva envolvente de resistencia lateral de entrepiso de un edificio hipotético de cuatro niveles de mampostería confinada que satisface todos los requisitos para que sea aplicable el Método Simplificado de diseño. Tiene las siguientes características: (1) La excentricidad estática en la dirección de análisis es la máxima permitida para aplicar el Método Simplificado. (2) El cortante resistente en la planta baja, en cada sentido del análisis, está lo más próximo posible al cortante actuante de diseño, y (3) las acciones serán las máximas esperadas según la NTCM-RCDF (2004).

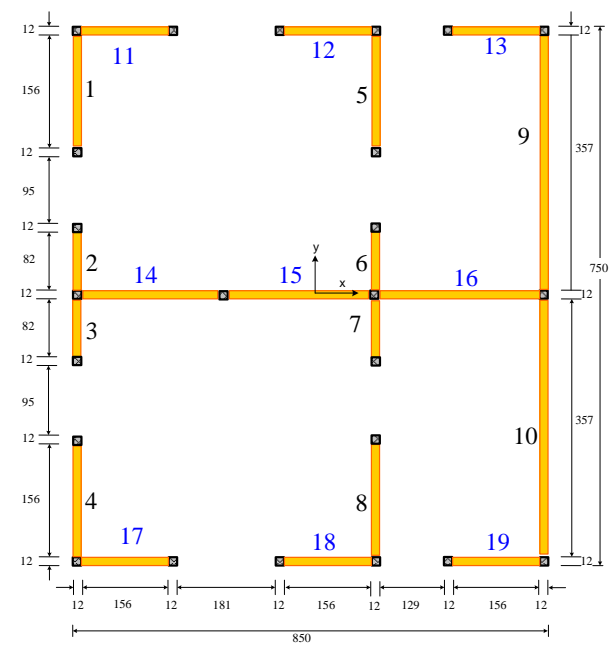

(a) Distribución en planta

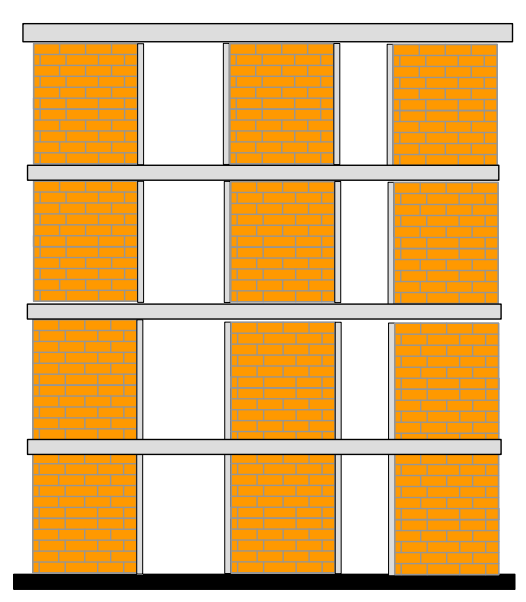

(b) Elevación en dirección X

Figura 23. Planta y elevación del edificio hipotético de mampostería

En la figura 23 se observa la distribución en planta de los muros y la elevación en la dirección X del edificio por analizar. En la dirección X el edificio presenta una excentricidad de $0.83 \mathrm{~m}$ que es el $97 \%$ del 
ancho en planta del edificio, siendo la excentricidad máxima permitida en esta dirección $0.85 \mathrm{~m}$. La altura de los entrepisos es $2.50 \mathrm{~m}$ y se considera que el edificio se ubica en la zona sísmica III del Valle de México. El sistema de piso está formado por una losa de $0.10 \mathrm{~m}$ de espesor y los muros tienen un espesor de $0.12 \mathrm{~m}$. El diseño se realizó tal que la mayoría de los muros de planta baja están esforzados al límite del cortante último actuante. En la tabla 3 se muestran las propiedades de los materiales utilizadas en este ejemplo.

Tabla 3. Propiedades de los materiales empleadas en el ejemplo hipotético

\begin{tabular}{|l|l|c|}
\hline Mampostería & \multicolumn{1}{|c|}{ Concreto } & Acero \\
\hline$f_{m}^{*}=20 \mathrm{MPa}$ & $f_{c}^{\prime}=15 \mathrm{MPa}$ & $f_{y}=420 \mathrm{MPa}$ \\
\hline$E_{m}=1200 \mathrm{MPa}$ & $E_{m}=18203 \mathrm{MPa}$ & $E_{s}=200000 \mathrm{MPa}$ \\
\hline$G_{m}=480 \mathrm{MPa}$ & & \\
\hline$v_{m}^{*}=0.35 \mathrm{MPa}$ & & \\
\hline
\end{tabular}

Para obtener el esfuerzo a compresión actuante en cada muro se empleó el criterio de áreas tributarias, considerando un peso de muro de $580 \mathrm{kgf} / \mathrm{m}$ y en la azotea una carga de servicio de $620 \mathrm{kgf} / \mathrm{m}^{2}$ y en los niveles inferiores de $633 \mathrm{kgf} / \mathrm{m}^{2}$. Los valores de las masa concentradas en cada entrepiso se muestran en la Tabla 4.

Tabla 4. Masa de cada entrepiso en el ejemplo hipotético

\begin{tabular}{|l|l|}
\hline Nivel & Masa kg \\
\hline 4 & 48856 \\
\hline 3 & 59039 \\
\hline 2 & 59039 \\
\hline 1 & 59039 \\
\hline
\end{tabular}

En el inicio del proceso del cálculo, la matriz de rigidez en la dirección de análisis X es:

$k_{1}=\left[\begin{array}{cccc}648.82 & -324.41 & 0 & 0 \\ -324.41 & 648.82 & -324.41 & 0 \\ 0 & -324.41 & 648.82 & -324.41 \\ 0 & 0 & -324.41 & 324.41\end{array}\right] \mathrm{kN}$

y en la dirección Y,

$k_{1}=\left[\begin{array}{cccc}616.144 & -308.072 & 0 & 0 \\ -308.072 & 616.144 & -308.072 & 0 \\ 0 & -308.072 & 616.144 & -308.072 \\ 0 & 0 & -308.072 & 308.072\end{array}\right] \frac{\mathrm{kN}}{\mathrm{mm}}$

En la figura 24 se muestran las curvas envolventes de resistencia de la planta baja del edificio en consideración. Para su elaboración se utilizó: 


$$
G_{m}=0.40 E_{m} \mathrm{y} v_{m}^{*}=35 \mathrm{MPa}
$$

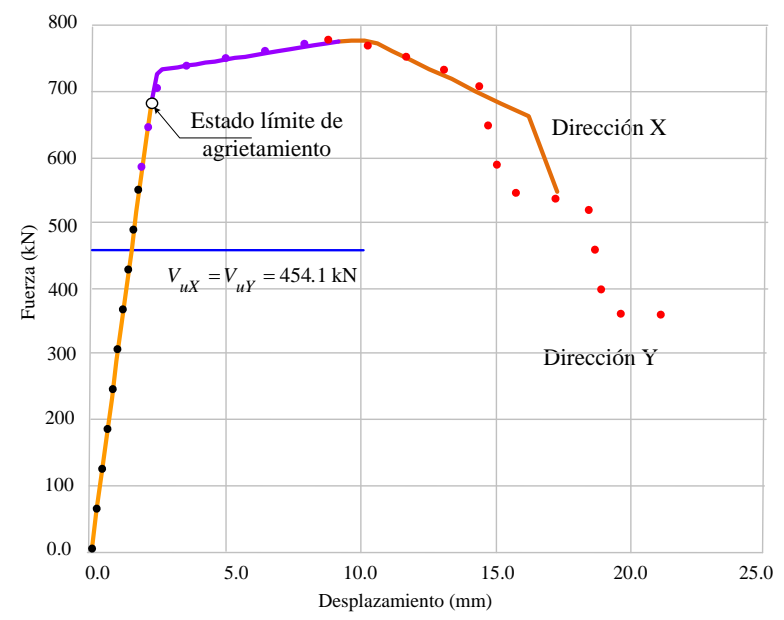

Figura 24. Envolventes de resistencia en las direcciones X y Y, del edificio hipotético

Las curvas envolventes, en ambos sentidos, predicen un cortante de agrietamiento mayor que el cortante actuante de diseño: $V_{u X}=V_{u Y}=454.10 \mathrm{kN}$, por lo que se espera que los muros permanezcan en el intervalo elástico. Además, las deformaciones por torsión hacen que en la dirección Y se tenga una resistencia máxima ligeramente mayor que en $X$, a pesar de que la densidad de muros es menor. En la figura 25 se muestra la evolución de la forma modal del primer modo de vibrar y el mecanismo de falla del denominado piso débil. Esto concuerda con lo observado experimentalmente y también con lo ocurrido después de un sismo en este tipo de estructuras.
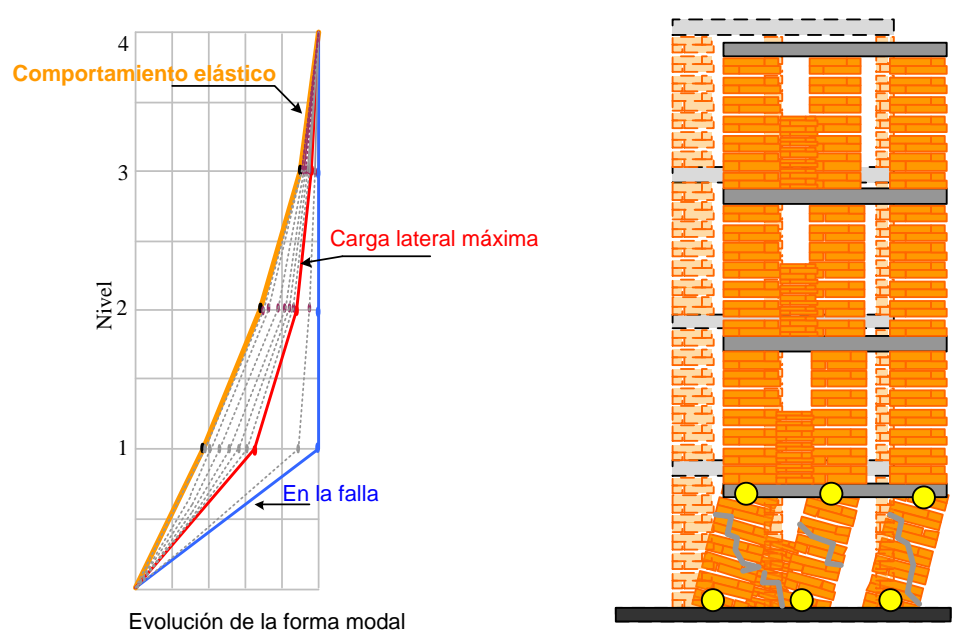

Figura 25. Evolución de la forma modal y mecanismo de falla

La metodología de incrementar los desplazamientos y no las cargas laterales, resultó estable, evitándose problemas de inestabilidad numérica al invertir la matriz de rigidez en cada ciclo del proceso. Una ventaja que presenta la metodología propuesta es que se puede determinar el estado de los muros que pertenecen a un entrepiso en particular, tal y como se muestra en la figura 26. En la figura 26a se muestran las curvas envolventes del entrepiso 1 y 2 ; en la figura $26 \mathrm{~b}$ las curvas envolventes del entrepiso 1 y 3 , y 
finalmente en la figura 26c las curvas del entrepiso del nivel 1 y 4 . Se puede ver que el cortante de agrietamiento disminuye en las envolventes de los pisos superiores, debido a que su capacidad de resistencia lateral es menor que la de los muros del primer nivel por tener menor esfuerzo a compresión. Cuando se cumple alguno de los criterios de falla en el nivel 1, el segundo nivel presenta una envolvente que se aproxima a la carga lateral máxima. Esto es congruente con la evidencia experimental en edificios con baja altura (Tomazevic y Klemenc, 1997).

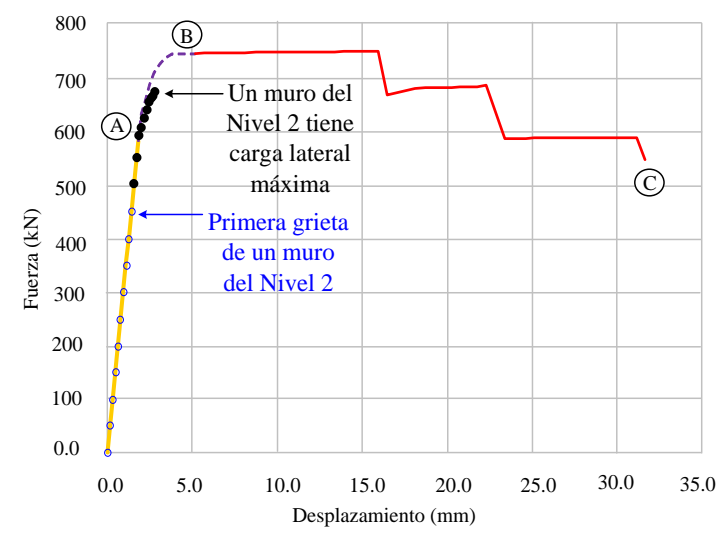

(a) Envolvente de resistencia, Nivel 1 y 2

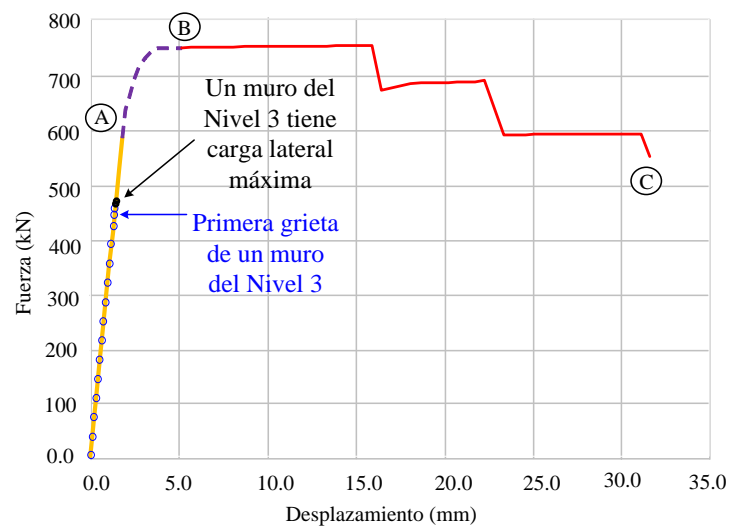

(b) Envolvente de resistencia, Nivel 1 y 3

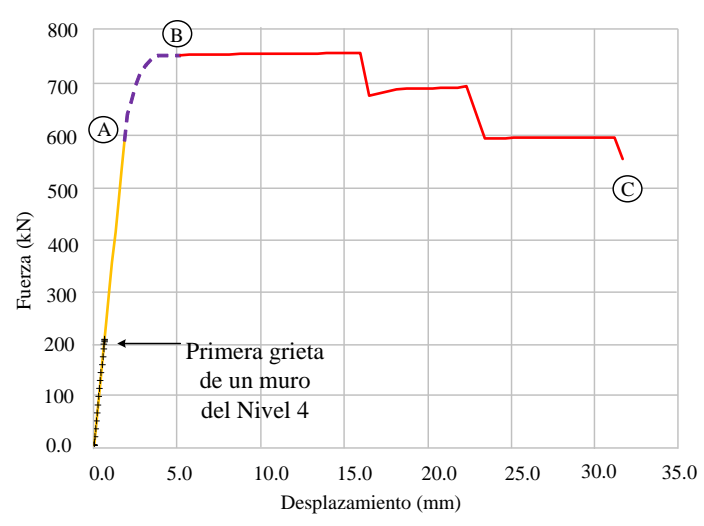

(c) Envolvente de resistencia, Nivel 1 y 4

Figura 26. Envolventes de resistencia lateral de los diferentes niveles

Otro beneficio de este tipo de análisis es que permite determinar el estado de un muro en particular, para cierta fuerza cortante lateral. Como ejemplo, en las figuras 27 a a la $27 \mathrm{c}$, se muestran las curvas envolventes de resistencia lateral de los muros 1, 2 y 10 de la planta baja del edificio (figura 23a), además, se indica el punto en el que el nivel 1 de la estructura alcanza el cortante de diseño. En la figura $27 \mathrm{~d}$ se muestran las envolventes de resistencia lateral del muro No. 1 ubicado en los diferentes niveles de la estructura. Así se pueden determinar el estado límite de comportamiento que tiene cada muro en particular y el estado de daño asociado. 


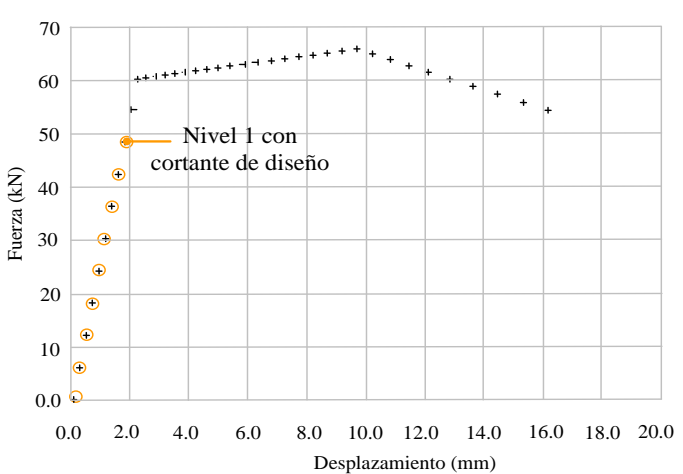

(a) Envolvente de resistencia, muro No 1

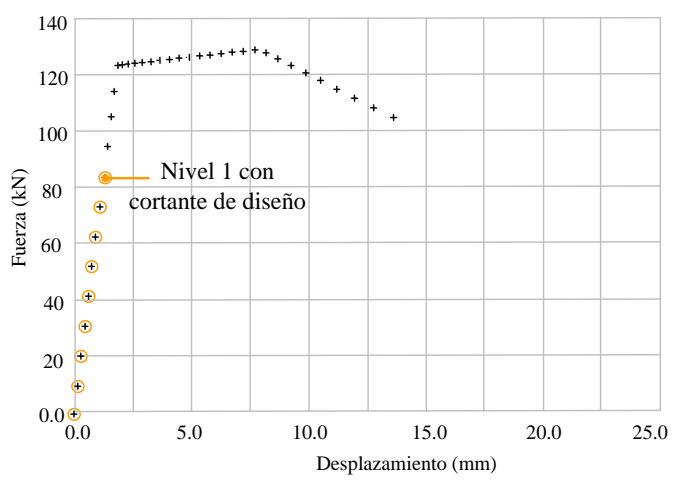

(c) Envolvente de resistencia, muro No 10

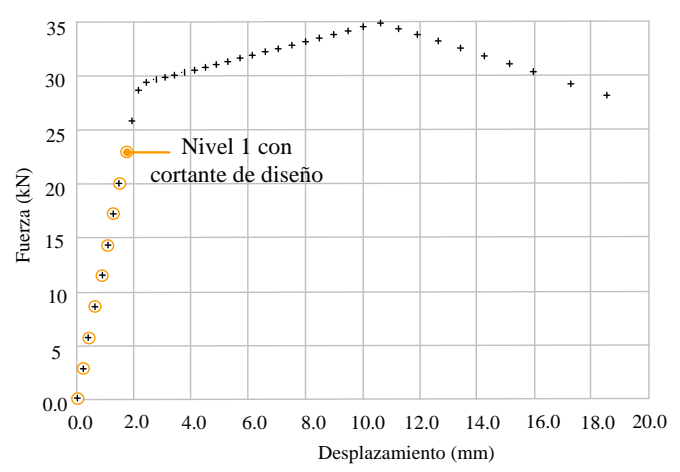

(b) Envolvente de resistencia, muro No 2

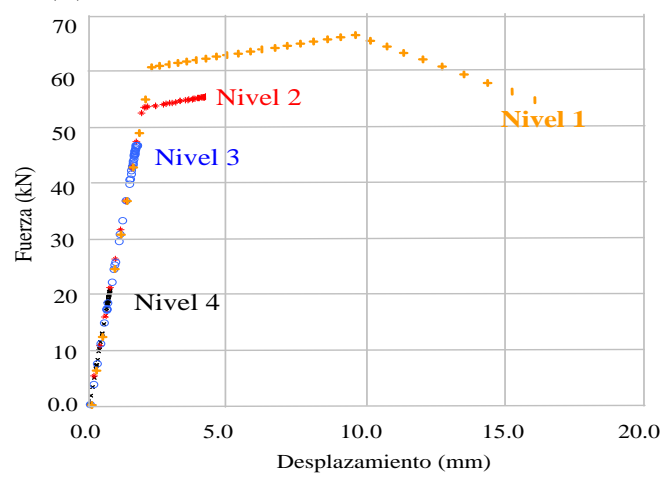

(d) Estado del muro No. 1, en cada nivel

Figura 27. Comparación de envolventes de resistencia lateral

En la Tabla 5 se muestran los valores de ductilidad y del factor de comportamiento sísmico calculados para este ejemplo. Se observa que el $Q$ en ambas direcciones de análisis se mantiene dentro del límite especificado de 2 (NTCM-RCDF, 2004). Si se considera un edificio de mampostería con 5 niveles y con altura de entrepiso de $2.50 \mathrm{~m}$, como límite de aplicación del Método Simplificado de diseño, la ductilidad de agrietamiento, $\mu_{1}$, debe ser 6 para que el edificio tenga un $Q=2$. En la Tabal 3 se puede ver que los valores de dicha ductilidad se acercan a dicho valor.

Tabla 5. Valores de ductilidad calculada analíticamente

\begin{tabular}{|c|c|c|c|}
\hline MODELO & $\mu_{1}$ & $\mu_{u}$ & $Q$ \\
\hline Dirección X & 6.53 & 3.07 & 2.27 \\
Dirección Y & 5.93 & 2.85 & 2.17 \\
\hline
\end{tabular}

\section{CONCLUSIONES}

Para evaluar el comportamiento sísmico de una estructura de mampostería es necesario realizar pruebas experimentales y desarrollar procedimientos analíticos que reproduzcan los resultados experimentales para su aplicación en la práctica ingenieril. En este trabajo se propone un método para el cálculo de la curva envolvente de resistencia lateral de entrepiso de estructuras de mampostería confinada con baja o mediana altura, que cumplen con los requisitos básicos para que sea aplicable el Método Simplificado de diseño (NTCM-RCDF, 2004). El procedimiento consiste en incrementar desplazamientos 
laterales, suponiendo un comportamiento fundamental en el primer modo de vibrar de la estructura. La curva envolvente idealizada se basa en resultados experimentales disponibles en México. Las curvas envolventes analíticas se compararon con las obtenidas experimentalmente en modelos ensayados bajo carga cíclica reversible y en la mesa vibradora del II-UNAM. Los resultados indican una adecuada predicción de la rigidez elástica, de la carga de resistencia asociada al primer agrietamiento y al estado de resistencia máxima; en algunos casos también fue posible predecir el desplazamiento asociado a cada punto anterior. El método propuesto permite calcular la ductilidad global y la de entrepiso, así como del factor de comportamiento sísmico referido en la Norma Técnica Complementaria al Reglamento de Diseño del Distrito Federal. La metodología se aplicó a un edificio de mampostería confinada con cuatro niveles cuyos muros se encuentran esforzados al límite máximo permitido por la reglamentación vigente en el Distrito Federal. Los resultados muestran un adecuado comportamiento sísmico de este tipo de estructuras, cuantificado por el factor del comportamiento sísmico y el valor de la ductilidad calculada.

\section{AGRADECIMIENTOS}

El segundo autor desea agradecer al Instituto de Ingeniería de la UNAM y en particular al Dr. Sergio Alcocer M. por su apoyo en la realización de este trabajo mediante la estancia en el laboratorio de la Mesa Vibradora de la UNAM. De igual manera se desea agradecer a la Universidad Michoacana de San Nicolás de Hidalgo, UMSNH, y al Ing. Iván Cornejo Vásquez por el apoyo otorgado para finalizar el trabajo.

\section{REFERENCIAS}

Anónimo (1999), "Edificaciones de Mampostería para Vivienda", Fundación ICA AC, México, DF, ISBN 968-7508-65-5.

Alcocer S M, J Arias y L Flores (2004), "Some developments on performance-based seismic design of masonry structures", Performance Based Seismic Design Concepts and Implementation, Proceeding of an International Workshop, Bled, Slovenia, June 28, PEER 2004/05.

Arias, A J (2005), "Ensayos en mesa vibradora de un modelo a escala 1:2 de edificio de mampostería confinada de tres niveles", Tesis de Maestría, Universidad Nacional Autónoma de México.

Astroza, M y A Schmidt (2004), "Capacidad de deformación de Muros de Albañilería Confinada para Distintos Niveles de Desempeño", Revista de Ingeniería Sísmica, Vol. 70, pp. 59-75.

Barragán, T R (2005), "Ensaye de una vivienda a escala de dos niveles de mampostería confinada", Tesis de Maestría, Universidad Nacional Autónoma de México.

Bazán, E (1980), "Muros de mampostería ante Cargas Laterales Estudios Analíticos", Tesis Doctoral, Facultad de Ingeniería, UNAM.

Beyer, K, A Dazio y M Priestley (2008), "Inelastic Wide-column Models for U-Shaped Reinforced Concrete Walls", Journal of Earthquake Engineering, Taylor and Francis, pp. 1-33.

Chipol, A (2001), "Estudio de la respuesta sísmica de modelos tridimensionales de edificios torsionalmente acoplados", Tesis de Maestría, Programa de Maestría y Doctorado en Ingeniería, UNAM.

Chopra, A K (1995), Dynamics of Structures, Theory and Applications to Earthquake Engineering, Prentice Hall, Inc., ISBN 0-13-855214-2. 
Chrysostomou, C Z, P Gergely y J F Abel (1992), "Nonlinear Seismic Response of Infilled Frames", Memorias X Congreso Mundial de Ingeniería Sísmica, Madrid, España, pp. 4435-4437.

Coral, M (2004), "Revisión de algunas de las hipótesis del método simplificado de análisis sísmico para muros de mampostería confinada", Tesis de Maestría, Programa de Maestría y Doctorado en Ingeniería, UNAM.

Crisafulli, F (1997), "Seismic Behaviour of Reinforced Concrete Structures with Masonry Infills", Tesis de Doctorado, University of Canterbury.

Fischinger M, D Berg, T Isakovic, M Tomazevic y R Zarnic (2004), "Performance based assessment from general Methodologies to specific implementations", Performance Based Seismic Design Concepts and Implementation, Proceeding of an International Workshop, Bled, Slovenia, June 28, PEER 2004/05.

Flores, L (1995), "Estudio analítico de estructuras de mampostería confinada", Tesis de Licenciatura, Facultad de Ingeniería de la UNAM.

Flores, L y S M Alcocer (2001), "Estudio Analítico de Estructuras de Mampostería confinada”, Informe de tesis de maestría, Centro Nacional de Prevención de Desastres, CENAPRED.

Holmes, M (1961), "Steel Frames with brickwork and concrete Infills", Proceeding of Instn. Civil Engineering, Vol. 19, pp. 473-478.

Ishibashi, K y H Kastumata (1994), “A Study on Nonlinear Finite Element Analysis of Confined Masonry Walls", Cuaderno de Investigación No. 15, CENAPRED.

Jean, R y F Cesín (2000), "Recomendaciones para el Diseño y Construcción de Estructuras de Mampostería", Primer Simposio Nacional de Estructuras de Mampostería para la Vivienda, México.

Kadir, M R (1974), "The Structural Behaviour of Masonry Infill Panel in Framed Structures", Tesis de Doctorado, Universidad de Edimburgo, Gran Bretaña.

Madan, A, A M Reinhorn, J B Mander y R E Valles (1997), "Modeling of Masonry Infill Panels for Structural Analysis”, Journal of Structural Engineering, ASCE, pp. 1295-1302.

Meli, R (1979), "Comportamiento Sísmico de Muros de Mampostería”, Reporte No. 352, Serie del Instituto de Ingeniería, Universidad Nacional Autónoma de México.

Moroni, M, M Astroza y S Tavonatti (1994), "Nolinear models for shear failure in confined masonry walls", The Masonry Soc. J., No. 12, Vol. 2, pp. 72-78.

Mosalam, K M, P Gregely, R N White y G Ayala (1996), "Effect of Geometrical configuration on the Seismic Response of Infilled Frames", Memorias de la XI Conferencia Mundial de Ingeniería Sísmica, Acapulco, México, CDROM, artículo 1675.

Newmark, N M y W Y Hall (1982), Earthquake Spectra and Design, Earthquake Engineering Research Institute.

NTCM-RCDF (2004) "Normas Técnicas Complementarias para diseño y construcción de Estructuras de Mampostería, Reglamento de Construcciones del Distrito Federal", Gaceta Oficial del Distrito Federal, marzo.

NTCS-RCDF (2004) "Normas Técnicas Complementarias para Diseño por Sismo, Reglamento de Construcciones del Distrito Federal", Gaceta Oficial del Distrito Federal, Tomo II, No. 103-BIS, octubre. 
Paulay, T, y M J N Priestley (1992), Seismic Design of Reinforced Concrete and Masonry Buildings, John Wiley and Sons, Inc. ISBN 0-471-54915.

Priestley, M J N, F Seible y G M Calvi (1996), Seismic Design and Retrofit of Bridges, John Wiley and Sons, Inc. ISBN 0-471-57998-X.

Sánchez, T A (1998), "Comportamiento de estructuras de mampostería confinada sujetas a cargas laterales", Tesis de Maestría, DEPFI-UNAM, Universidad Nacional Autónoma de México.

Sánchez, S, R Arroyo y S Jerez (2010), "Modelo de un grado de libertad para evaluar la curva carga lateral-distorsión en muros de mampostería confinada", Revista de Ingeniería Sísmica, No. 83, pp. 25-42.

Siete, B J (2011), "Fragilidad basada en desplazamiento para edificaciones de mampostería confinada", Tesis de Maestría, Universidad Michoacana de San Nicolás de Hidalgo, Morelia, Michoacán.

Tena, A, J Cano, A López y M A Pérez (2010), "Revisión y actualización del Método Simplificado de Análisis de Estructuras de mampostería de los Reglamentos de diseño sísmico de México", Revista de Ingeniería Sísmica, Vol. 82, pp. 1-34.

Tomazevic, M (1999), Earthquake-Resistant Design of Masonry Buildings, Series on Innovation in Structures and Construction, Vol. 1. Imperial College Press, ISBN 1-86094-066-8.

Tomazevic, M y I Klemenc (1997a), "Verification of Seismic Resistance of Confined Masonry Buildings", Earthquake Engineering and Structural Dynamics, Vol. 26, pp. 1073-1088.

Tomazevic, M y I Klemenc (1997b), "Seismic Behaviour of Confined Masonry Walls", Earthquake Engineering and Structural Dynamics, Vol. 26, pp. 1059-1071.

Tomazevic, M, V Bosiljkov y P Weiss (2004), "Structural Behavior Factor for masonry structures", $13^{\text {th }}$ World Conference on Earthquake Engineering, Vancouver, B. C., Canada, August, paper No. 2642.

UNIANDES (2001), "Comportamiento sísmico de muros de mampostería confinada", Asociación Colombiana de Ingeniería Sísmica.

Vázquez, V A (2005), "Ensaye experimental de viviendas de mampostería confinada de un piso mediante el ensaye en mesa vibradora", Tesis de Maestría, Universidad Nacional Autónoma de México.

Valles, R E, A M Reinhorn, S K Kunnath, C Li y A Madan (1996), “IDARC 2D, version 4”, National Center for Earthquake Engineering Research, State University of New York at Buffalo.

Zúñiga, O y A Terán (2008), "Evaluación basada en desplazamientos de edificaciones de mampostería confinada", Revista de Ingeniería Sísmica, Vol. 79, pp. 25-48. 